\title{
Effects of detuning, gain-guiding, and index antiguiding on the dynamics of two laterally coupled semiconductor lasers
}

\author{
M. J. Adams, ${ }^{1,{ }^{*}}$ Nianqiang Li, ${ }^{1}$ B. R. Cemlyn, ${ }^{1}$ H. Susanto, ${ }^{2}$ and I. D. Henning ${ }^{1}$ \\ ${ }^{1}$ School of Computer Science and Electronic Engineering, University of Essex, Wivenhoe Park, Colchester CO4 3SQ, United Kingdom \\ ${ }^{2}$ Department of Mathematical Sciences, University of Essex, Wivenhoe Park, Colchester CO4 3SQ, United Kingdom
}

(Received 8 February 2017; published 30 May 2017)

\begin{abstract}
Four examples of laterally coupled semiconductor lasers with different waveguiding structures have been studied using coupled mode theory and allowing for frequency detuning between the lasers. The structures include purely real index guiding, pure gain-guiding, and combinations of index guiding and antiguiding with gain-guiding. The dynamics of these four systems have been explored using AUTO software (standard numerical continuation package), linear stability analysis, and direct integration of the rate equations. Convincing agreement between results obtained by these three methods has been demonstrated, including effects due to variation of laser pumping rate, detuning, and linewidth enhancement factor. A periodicity of behavior with laser separation has been revealed that was previously overlooked. This periodicity has increasing influence on the bifurcations of the system as the structures develop from those with purely real guidance to a combination of index antiguiding and gain-guiding. The laser design and operating parameters used are realistic for a wide range of edge-emitting and surface-emitting lasers of practical importance, so that the dynamics studied here are relevant to real systems of coupled lasers.
\end{abstract}

DOI: 10.1103/PhysRevA.95.053869

\section{INTRODUCTION}

Arrays of evanescently coupled semiconductor lasers have been of interest since the first experimental report in 1970 [1]. In applications requiring high-power sources with diffractionlimited single-lobe output beams, phase-locked arrays of edge-emitting lasers (EELs) have proved effective [2,3]. Theoretical treatments of these one-dimensional arrays are usually based on either coupled-mode theory or normal-mode (sometimes also termed "supermode" or "composite-cavity mode") analysis; a comparison of these methods has been presented in [4]. It is well known that coupled-mode theory does not apply to strongly coupled laser arrays or to structures with leaky-wave array modes that are important for high-power source design. Hence more rigorous modal analysis is used for two-dimensional arrays of vertical-cavity surface-emitting lasers (VCSELs) such as those for operation at telecom wavelengths [5], or for more advanced EEL configurations such as quantum cascade laser arrays for the mid-infrared spectral region [6]. Again, in all cases the emphasis has been on developing designs and implementing appropriate technology to obtain stable high-power operation in a narrow beam.

Early theoretical analysis of the dynamics of coupled EELs showed that individual array elements could exhibit instability with large-amplitude chaotic pulsations even though the total output of the array would settle to a quasisteady state [7]. For a two-element array, conditions for the stability of the in-phase and out-of-phase solutions were derived in terms of the coupling strength, excess pump current, linewidth enhancement factor, and the ratio of carrier recombination lifetime to photon lifetime [8]. A study of the oscillation frequency of the output showed that for low coupling strength this is dominated by the relaxation oscillation frequency, while for stronger coupling the frequency is dominated by the rate of

\footnotetext{
*adamm@essex.ac.uk
}

energy transfer between the lasers governed by the coupling coefficient [8]. Of course, for sufficiently strong coupling the coupled-mode theory is no longer accurate and a normal-mode treatment is more appropriate. For example, a study of the interaction between two optically pumped VCSELs emitting at $880 \mathrm{~nm}$ showed that coupled-mode theory was valid for separations greater than or equal to $13 \mu \mathrm{m}$ [9].

In $[7,8]$ the coupling coefficient was assumed to be a real number, as determined by conventional coupled-mode theory (see, e.g., [10]). However, in many cases of interest account must be taken of the effects of gain-guiding and carrier-induced index antiguiding that are known to be important in semiconductor lasers with little or no built-in lateral optical confinement. In these cases the coupling coefficient must be complex and the coupled-mode approach must be modified accordingly [11-14]. It has also been shown that complex coupling coefficients are required in order to account for experimental measurements of instabilities in coupled microchip lasers [15], while the special case of purely imaginary coupling coefficient has been used to model experiments on a $\mathrm{Nd}$ :YAG laser crystal pumped with beams from two optical fibres [16]. In all these theoretical treatments the optical cavity resonances of the two lasers were assumed to be identical. However, in reality small variations in cavity structure (width, length, material properties, etc.) will lead to a difference in the cavity resonances. This detuning effect has been included in modeling the dynamics of two laterally coupled EELs [17], although the (purely real) coupling coefficient in this case was calculated using the concept of a laser with a transmitting interface [18,19], rather than the conventional coupling via evanescent fields. The detuning has also been included in a model of the coupling between two evanescently coupled, optically pumped Nd:YAG lasers [20]; in this case the detuning was controlled by including a foursecond wedge angle between the two end surfaces of the YAG etalon and rotating the etalon about the axis of propagation.

In this contribution we present a study of the dynamics in two laterally coupled semiconductor lasers including the 
effects of gain-guiding and carrier-induced index antiguiding via a complex coupling coefficient as well as the effects of detuning between the cavity resonances. This approach generalizes the treatments of EELs in [7,8,11-14,17,21,22] as well as the work on solid state lasers for which there is no nonlinearity due to carrier-induced effects $[15,16,20]$; i.e., the linewidth enhancement factor as used in semiconductor laser theory is zero. Time-dependent coupled-mode equations are used with the coupling coefficient calculated from a simple model of slab waveguides with complex permittivity. Bifurcation analysis is used to reveal regions of stable phase-locking, periodic, and chaotic behavior. Results are presented as functions of laser separation for a number of different optical confinement scenarios related to different EEL or VCSEL structures.

The paper is organized as follows. Section II summarizes the coupled-mode treatment of two parallel slab waveguides allowing for a complex dielectric permittivity in all regions, i.e., with gain and loss in the waveguide cores, and loss in the claddings. Section III gives the rate equations for the system in normalized variables and also the numerical values selected for the key parameters of the four different waveguide structures to be studied. In Sec. IV a small-signal analysis of the rate equations is presented, leading to simple approximate expressions for the stability boundaries as functions of the coupling coefficient, frequency detuning, pumping rate, and other relevant parameters. Section V presents our results for the dynamics of the four sets of coupled lasers and compares those obtained from numerical bifurcation analysis with those from direct numerical integration of the rate equations and from the approximations derived in Sec. IV. In the final section we summarize our conclusions, while mathematical details of the derivation of the coupled-mode rate equations and of the small-signal analysis are given in two appendices.

\section{COUPLED SLAB WAVEGUIDES}

We consider two identical laser waveguides, A and B, each of width $2 a$, with an edge-to-edge separation of $2 d$, as illustrated schematically in Fig. 1.

The complex dielectric permittivity, real refractive index, and gain per unit length in each of the waveguide cores are $\varepsilon_{1}$, $n_{1}$, and $g$, respectively, and the permittivity and refractive index elsewhere are $\varepsilon_{2}, n_{2}$. The background attenuation coefficient per unit length due to effects such as scattering and intervalence band absorption in all regions is $\alpha$. It follows that

$$
\varepsilon_{1}=\left(n_{1}+i \frac{g-\alpha}{2 k}\right)^{2}, \quad \varepsilon_{2}=\left(n_{2}-i \frac{\alpha}{2 k}\right)^{2}
$$

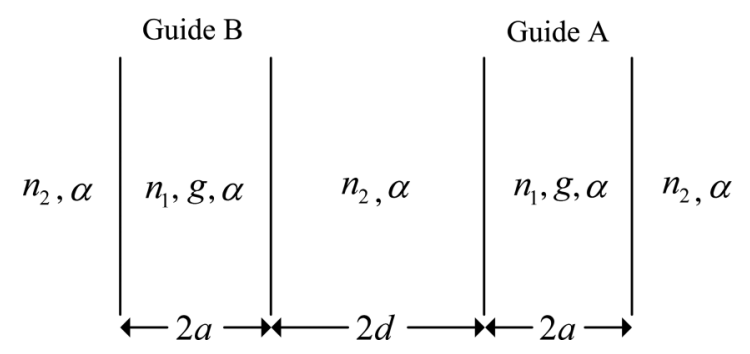

FIG. 1. Schematic of coupled slab waveguides. where $k$ is the free-space wave number. The complex normalized frequency $V$ is defined as

$$
V^{2}=a^{2} k^{2}\left(\varepsilon_{1}-\varepsilon_{2}\right) \text {. }
$$

For the case when the gain and loss are neglected, the definition in Eq. (2) reduces to the conventional version for real step-index dielectric waveguides $v^{2}=a^{2} k^{2}\left(n_{1}^{2}-n_{2}^{2}\right)$, where the symbol $v$ has been used for the real normalized frequency.

Defining $\Delta n=n_{1}-n_{2}$ and $n \cong n_{1}, n_{2}$, for the usual situation that $\Delta n, g / k, \alpha / k \ll n_{1}, n_{2}$, it follows from Eq. (1) that to a very good approximation Eq. (2) can be written as

$$
V^{2} \cong a^{2} k^{2} 2 n\left(\Delta n+i \frac{g}{2 k}\right) .
$$

Equation (3) is similar to the approximation used in [23] with the differences that (a) in [23] a loss was only assumed in the cladding regions, not in the waveguide core, and (b) in [23] the definition of gain used was that for the field amplitude, whereas here it is the gain of the intensity and thus differs by a factor of 2 .

The expressions for the spatial dependence $\Psi_{A}(x)$ of the fields of the even-order transverse electric (TE) modes in guide A are given (with the origin of $x$ at the center of guide A) by

$$
\begin{aligned}
& \Psi_{A}=\cos \left(U \frac{x}{a}\right), \quad|x| \leqslant a, \\
& \Psi_{A}=\cos (U) \exp \left(-W \frac{|x|-a}{a}\right), \quad|x| \geqslant a,
\end{aligned}
$$

where $U^{2}+W^{2}=V^{2}$. Similar expressions apply for the corresponding case of guide B. Solutions for complex $U$ and $W$ are found from the eigenvalue equation which assures continuity of the electric field and its gradient at $|x|=a$ :

$$
\begin{aligned}
& \tan (U)=\frac{W}{U}, \text { or, equivalently, } \\
& \sin (U)=\frac{W}{V} \text { or } \cos (U)=\frac{U}{V} .
\end{aligned}
$$

The corresponding eigenvalue equation for odd-order TE modes is

$$
\begin{aligned}
& \tan (U)=-\frac{U}{W}, \text { or, equivalently, } \\
& \sin (U)=-\frac{U}{V} \text { or } \cos (U)=\frac{W}{V} .
\end{aligned}
$$

The condition for a propagating mode is given by $W_{r}>0$, where $W$ is expressed in terms of real and imaginary parts, $W_{r}$ and $W_{i}$, respectively. The case of real index guiding is well known and the solutions of Eqs. (5) and (6) (written with lowercase $u, v, w$ according to the conventional notation mentioned above) are presented in many textbooks; the waveguide is single mode (i.e., the lowest even-order mode is the only one permitted) from $v=0$ to $v=\pi / 2$, which is the cutoff for the first odd-order mode. The case of pure gain guiding ( $\Delta n=0)$ was considered first by Schlosser [24] who calculated solutions of Eqs. (5) and (6) and showed that the waveguide is single mode for $|V| \leqslant 1.877$. For the general case of complex permittivities, it is worth noting that, in contrast to the case of purely real symmetric slab guides, the fundamental 
mode can have a nonzero cutoff. The cutoff curves for several even- and odd-order modes have been presented by Buus [25] in plots of $|V|$ versus $\arg (V)$ and by Siegman [23] in plots of $\operatorname{Im}\left(V^{2}\right)$ versus $\operatorname{Re}\left(V^{2}\right)$.

The solutions of the solitary waveguides A and B as discussed above are used in coupled-mode theory to describe the interactions between the guides. Specifically, two quantities must be calculated, namely the (real) optical confinement factor $\Gamma$ and the (complex) coupling coefficient $\eta$, whose definitions (see Appendix A) are

$$
\begin{gathered}
\Gamma=\frac{I_{1}}{I_{2}} \\
\eta=\frac{c k}{n}\left(\Delta n+i \frac{g}{2 k}\right) \frac{I_{3}}{I_{2}} \exp \left(-2 W \frac{d}{a}\right),
\end{gathered}
$$

where the integrals are given, using the expressions from Eq. (4), by

$$
\begin{aligned}
& I_{1}=2 \int_{0}^{a}\left|\cos \left(U \frac{x}{a}\right)\right|^{2} d x \\
& I_{2}=I_{1}+2|\cos (U)|^{2} e^{2 W_{r}} \int_{a}^{\infty} e^{-2 W_{r} x / a} d x, \\
& I_{3}=\cos (U) e^{-W} \int_{-a}^{a} \cos \left(U^{*} \frac{x}{a}\right) e^{-W x / a} d x .
\end{aligned}
$$

In general these integrals must be evaluated numerically. For antiguides $(\Delta n<0)$ an analytical approximation for $\Gamma$ has been presented by Buus and Botez [26]. For the case of the lowest-order mode, an exact algebraic expression for $\Gamma$ has been presented by Siegman [23]:

$$
\Gamma=\frac{\left(U_{r} \sin 2 U_{r}-U_{i} \sinh 2 U_{i}\right)\left(U_{i} \sin 2 U_{r}+U_{r} \sinh 2 U_{i}\right)}{2 U_{r} U_{i}+2 U_{r} U_{i} \cos 2 U_{r} \cosh 2 U_{i}+\left(U_{r}^{2}-U_{i}^{2}\right) \sin 2 U_{r} \sinh 2 U_{i}},
$$

where $U=U_{r}+i U_{i}$. For the case of real index guidance this simplifies to

$$
\Gamma=\frac{w\left(v^{2}+w\right)}{v^{2}(1+w)}
$$

The value of core gain $g$ to be used in Eq. (3) for $V^{2}$ is usually that at lasing threshold. The lasing threshold condition is given by

$$
\Gamma g_{t h}=\frac{n_{g}}{c \tau_{p}},
$$

where $\tau_{p}$ is the photon lifetime, $n_{g}$ is the group index, and $c$ is the speed of light. Since, in general, the calculation of $\Gamma$ requires a knowledge of $g$, some iteration is necessary to determine $(g, \Gamma)$ pairs that satisfy (12) for a given value of $\tau_{p}$.

The coupling coefficient for the case of real index guidance is also easily evaluated as

$$
\eta=\frac{c}{k n^{2}}\left(\frac{u w}{a v}\right)^{2} \frac{1}{(w+1)} \exp \left(-2 W \frac{d}{a}\right) .
$$

For the general case of complex permittivities, no algebraic expression has yet been presented for $\eta$, to the best of our knowledge. However, it is clear from Eq. (8) that the dependence of coupling on $d / a$ is explicit, so that the coefficient $\eta$ can be parametrized as

$$
|\eta|=C_{\eta} \exp \left(-2 W_{r} \frac{d}{a}\right), \quad \arg (\eta)=C_{\theta}-2 W_{i} \frac{d}{a},
$$

where $C_{\eta}, C_{\theta}$ can be found from numerical integration.

\section{RATE EQUATIONS AND KEY PARAMETERS}

The normalized rate equations that describe the coupled lasers (see Appendix A) are

$$
\frac{d Y_{A}}{d t}=\frac{1}{2 \tau_{p}}\left(M_{A}-1\right) Y_{A}-|\eta| Y_{B} \sin (\theta+\phi),
$$

$$
\begin{gathered}
\frac{d Y_{B}}{d t}=\frac{1}{2 \tau_{p}}\left(M_{B}-1\right) Y_{B}-|\eta| Y_{A} \sin (\theta-\phi), \\
\frac{d \phi}{d t}=\frac{\alpha_{H}}{2 \tau_{p}}\left(M_{A}-M_{B}\right)-\Delta \Omega \\
\quad+|\eta|\left[\frac{Y_{A}}{Y_{B}} \cos (\theta-\phi)-\frac{Y_{B}}{Y_{A}} \cos (\theta+\phi)\right], \\
\frac{d M_{A, B}}{d t}=\frac{1}{\tau_{N}}\left[Q_{A, B}-M_{A, B}\left(1+Y_{A, B}^{2}\right)\right],
\end{gathered}
$$

where $Y_{A}, Y_{B}$ are the normalized field amplitudes and $M_{A}$, $M_{B}$ are the normalized carrier densities in guides $\mathrm{A}, \mathrm{B}$, respectively, $\phi$ is the phase difference between the fields in $\mathrm{B}$ and $\mathrm{A}, \Delta \Omega$ is the detuning between the cavity resonances of lasers $\mathrm{B}$ and $\mathrm{A}, \tau_{N}$ is the carrier lifetime, $\tau_{p}$ is the photon lifetime, $\alpha_{H}$ is the linewidth enhancement factor, $|\eta|$ and $\theta$ are the amplitude and phase of the coupling coefficient, and $Q_{A}$, $Q_{B}$ are the normalized pumping rates in lasers $\mathrm{A}$ and $\mathrm{B}$.

Equations (15)-(18) can be compared with those used in [7,8,13-15,17,20-22]. Dealing first with the nonsemiconductor laser work in $[15,20]$, it should be noted that (a) the linewidth enhancement factor does not appear in the equations used, and (b) an interchange in notation of the real and imaginary parts of $\eta$ leads to an interchange of the sines and cosines in the equations corresponding to Eqs. (15)-(17) as they appear in [15]. The corresponding rate equations in [20] are written in terms of real and imaginary parts of $\eta=\eta_{r}+i \eta_{i}$, instead of using amplitude and phase as here. The latter notation is used also in analysis of semiconductor laser pairs in [13,14,21,22], although in those papers the detuning $\Delta \Omega$ is taken as zero. For reference, the corresponding forms of Eqs. (15)-(17) are

$$
\begin{aligned}
& \frac{d Y_{A}}{d t}=\frac{1}{2 \tau_{p}}\left(M_{A}-1\right) Y_{A}-Y_{B}\left(\eta_{r} \sin \phi+\eta_{i} \cos \phi\right), \\
& \frac{d Y_{B}}{d t}=\frac{1}{2 \tau_{p}}\left(M_{B}-1\right) Y_{B}+Y_{A}\left(\eta_{r} \sin \phi-\eta_{i} \cos \phi\right),
\end{aligned}
$$


TABLE I. Values of key parameters for modeling, using material parameter values given in text.

\begin{tabular}{lcccccccrrr}
\hline \hline$\Delta n$ & $g\left(\mathrm{~cm}^{-1}\right)$ & $g / 2 k$ & $\operatorname{Re}\left(V^{2}\right)$ & $\operatorname{Im}\left(V^{2}\right)$ & $\Gamma$ & $W_{r}$ & $W_{i}$ & $C_{Q}$ & $C_{\eta}\left(\mathrm{ns}^{-1}\right)$ & $C_{\theta}(\mathrm{rad})$ \\
\hline 0.0005 & 90.6 & 0.000937 & 1.27 & 2.38 & 0.817 & 1.09 & 0.896 & 11.0 & 90.2 \\
0 & 99.3 & 0.00103 & 0 & 2.61 & 0.745 & 0.795 & 1.22 & 10.1 & 9.233 \\
-0.0005 & 108 & 0.00112 & -1.27 & 2.84 & 0.685 & 0.604 & 1.61 & 9.26 & 96.3 & 0.294 \\
\hline \hline
\end{tabular}

$$
\begin{aligned}
\frac{d \phi}{d t}= & \frac{\alpha_{H}}{2 \tau_{p}}\left(M_{A}-M_{B}\right)-\Delta \Omega+\eta_{r} \cos \phi\left(\frac{Y_{A}}{Y_{B}}-\frac{Y_{B}}{Y_{A}}\right) \\
& +\eta_{i} \sin \phi\left(\frac{Y_{A}}{Y_{B}}+\frac{Y_{B}}{Y_{A}}\right) .
\end{aligned}
$$

When $\Delta \Omega=0$ these equations reproduce those used in $[13,14,21,22]$. In the case of $[7,8,17]$, the coupling coefficient is a real number, corresponding to the case $\eta_{i}=0$ in Eqs. (19)-(21), although of these papers only [17] includes the effect of detuning between the lasers. In fact the authors of [17] also compared the results of the coupled-mode theory for dynamic behavior of a two-element laser array with those of a composite-cavity mode analysis and with those from a so-called "individual-laser theory" where the coupling coefficient $\eta$, as defined here, is replaced by one defined as $\kappa=i \eta$. For the former comparison the agreement was very good provided that the coupling was not too strong. However, the latter comparison did not give agreement and this was attributed to the failure of the "individual-laser theory" to satisfy the boundary conditions on the waveguide fields, as defined by Eqs. (5) and (6) above. Nevertheless, it is worth noting that this approach has been used in the literature (e.g., [27]) to model coupled semiconductor laser systems with the coupling coefficient $\kappa$ treated as a variable parameter.

The normalized rate equations use the three rate parameters $\eta, \tau_{N}$, and $\tau_{p}$, as well as the linewidth enhancement factor $\alpha_{H}$. For numerical work here we calculate $\eta$ from Eq. (14), together with the values $\tau_{N}=1.0 \mathrm{~ns}, \tau_{p}=1.53 \mathrm{ps}$, and $\alpha_{H}=2$ or 0 . The only other quantity that needs to be specified is the normalized pump rate $Q$, which is defined for the case of equal pumping in both lasers as

$$
Q=\frac{c}{n_{g}} \Gamma a_{\mathrm{diff}} \tau_{p}\left(P \tau_{N}-N_{o}\right),
$$

where $N_{o}$ is the transparency carrier concentration, $a_{\text {diff }}$ is the differential gain, and $P$ is the pumping rate with dimensions $L^{-3} T^{-1}$. The threshold value of pumping is given by

$$
P_{t h}=\frac{1}{\tau_{N}}\left(N_{o}+\frac{n_{g}}{\Gamma c a_{\mathrm{diff}} \tau_{p}}\right) .
$$

Hence it follows that $Q$ can be expressed in terms of $P / P_{t h}$ as

$$
Q=C_{Q}\left(\frac{P}{P_{t h}}-1\right)+\frac{P}{P_{t h}},
$$

where $C_{Q}=\frac{a_{\text {diff }}}{g_{t h}} N_{o}$.

For numerical work we use the values $a=4 \mu \mathrm{m}, k=$ $4.833 \mu \mathrm{m}^{-1}, a_{\text {diff }}=1 \times 10^{-15} \mathrm{~cm}^{2}, N_{o}=1 \times 10^{18} \mathrm{~cm}^{-3}$, and $n=3.4$. We restrict our analysis to the case where a solitary laser supports a single transverse mode. Thus we choose 3 values of the real refractive index difference $\Delta n$, namely 0 , \pm 0.0005 , and use these to analyze the dynamics for pairs of identical complex permittivity coupled lasers using Eq. (3) to define the complex normalized frequency with the value of core gain at threshold given by Eq. (12). The resultant set of calculated values for the key parameters is given in Table I.

The first line in Table I has positive index guiding of the type often encountered for example in buried heterostructure EELs or oxide-aperture VCSELs. However, when allowance is made for the effects of gain and loss, it is clear that the imaginary part of $V^{2}$ can be similar in magnitude or greater than the real part. The middle row is the case of no built-in index guiding, so that guidance only occurs because of the gain in the active region. The last row simulates index antiguiding (negative real part of $V^{2}$ ) with gain-guiding; this is the structure with the lowest optical confinement in the table.

We also consider separately the case of purely real index guiding, for comparison with similar work in the literature $[7,8,17]$. In this case, in order to achieve the maximum optical confinement while remaining single mode, we choose $v=\pi / 2\left(v^{2}=2.47\right)$. It follows from the solution of the eigenvalue equation that $w=1.26$, from Eq. (11) that $\Gamma=$ 0.844, that $C_{Q}=11.4$, and from Eqs. (13) and (14) that $C_{\eta}=83.6 \mathrm{~ns}^{-1}$ and $C_{\theta}=0$.

Figure 2 shows the variation of $\tau_{p} \eta_{r}$ and $\tau_{p} \eta_{i}$ with $d / a$ for the sets of parameters in Table I and the real index guide defined above. The range of $d / a$ is chosen to be from 1 to 6 , since this ensures that the coupling is sufficiently weak as to be a good approximation when compared to composite-cavity mode analysis [17]. We note in passing that all the values of $C_{\eta}$ and $C_{\theta}$ in Table I for various waveguide configurations are rather similar to those for the simple case of real index guiding; the main difference is that the phase of the coupling coefficient varies linearly with the spacing $2 d$ for the complex waveguides, as shown in Eq. (14). It follows that there is a periodic behavior of $\eta_{r}$ and $\eta_{i}$ with $d / a$, as seen in Figs. 2(b) $-2(d)$, and that the period is given by $\pi / W_{i}$.

While there have been reports of a change of sign of $\eta_{r}[12]$ and $\eta_{i}$ [13] with variation of spacing, in these cases the decay of $|\eta|$ was so rapid (owing to a large value of $W_{r}$ ) that the periodic behavior was not observed. Change of sign of both $\eta_{r}$ and $\eta_{i}$ with $d / a$ has been found in coupling calculations for pairs of gain-guided and index antiguided fibers [28] where the zero-crossing points are determined by the Bessel function zeros. As we shall see below, the periodic behavior of $\eta_{r}$ and $\eta_{i}$ with $d / a$ is important when we consider the implications for the dynamics of laterally coupled lasers.

\section{SMALL-SIGNAL ANALYSIS OF THE RATE EQUATIONS}

The steady-state solution of Eqs. (18)-(21) cannot be found analytically in general. However, for the range of parameter values used here, approximate solutions can be 

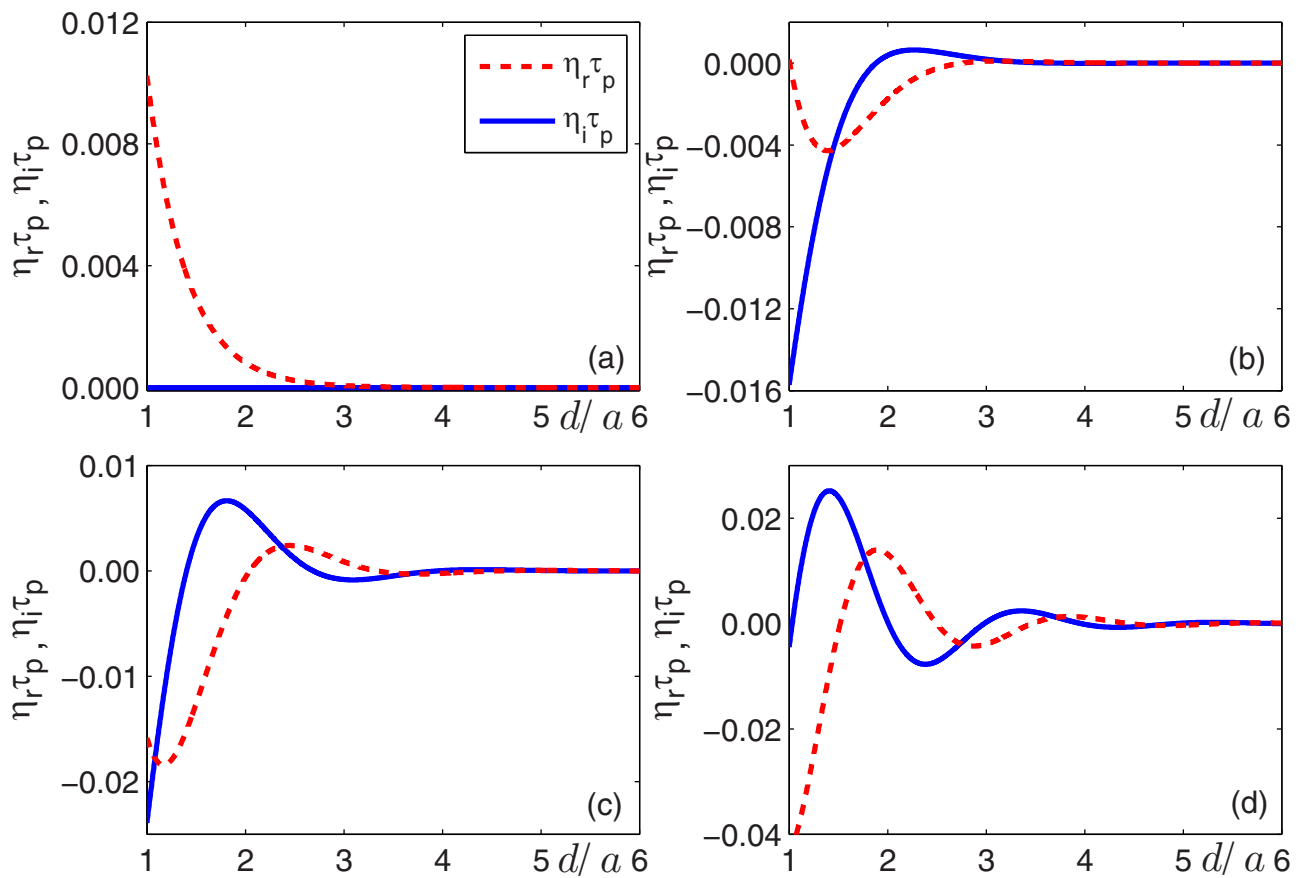

FIG. 2. Real (broken red) and imaginary (solid blue) parts of the product of coupling coefficient and photon lifetime versus $d / a$ for the cases (a) $\Delta n=0.000971$, (b) $\Delta n=0.0005$, (c) $\Delta n=0$, (d) $\Delta n=-0.0005$, with other parameters as in Table I and accompanying text.

found by noting that the values of $\tau_{p}|\eta|$ at $d / a=1$ vary from 0.010 (for $\Delta n=0.000971$, the strongest guide) to 0.044 (for $\Delta n=-0.0005$, the weakest guide). For values of $d / a>1$, the values of $\tau_{p}|\eta|$ decay exponentially as given by Eq. (14). Hence to an acceptable level of accuracy the steady-state solution of Eqs. (18)-(21) can be approximated to first order in small quantities $\tau_{p} \eta_{i}$ and $\tau_{p} \eta_{r}$. The results for the case of equal pumping in the lasers $\left(Q_{A}=Q_{B} \equiv Q\right)$ are

$$
\begin{gathered}
M_{A s} \cong 1+2 \tau_{p}\left(\eta_{r} \sin \phi_{s}+\eta_{i} \cos \phi_{s}\right), \\
M_{B s} \cong 1-2 \tau_{p}\left(\eta_{r} \sin \phi_{s}-\eta_{i} \cos \phi_{s}\right), \\
Y_{A s}^{2} \cong Q\left[1-2 \tau_{p}\left(\eta_{r} \sin \phi_{s}+\eta_{i} \cos \phi_{s}\right)\right]-1, \\
Y_{B s}^{2} \cong Q\left[1+2 \tau_{p}\left(\eta_{r} \sin \phi_{s}-\eta_{i} \cos \phi_{s}\right)\right]-1, \\
\sin \phi_{s} \cong \pm \frac{\Delta \Omega}{2\left(\eta_{i}+\alpha_{H} \eta_{r}\right)},
\end{gathered}
$$

where the subscript " $s$ " denotes steady state. Note that in the case of zero detuning $(\Delta \Omega=0)$, Eqs. (25)-(29) reduce to the well-known case of the in-phase $\left(\phi_{s}=0\right)$ and antiphase $\left(\phi_{s}=\pi\right)$ solutions.

Neglecting terms of order $\tau_{p} \eta_{i}$ and $\tau_{p} \eta_{r}$ in Eqs. (25)-(28) yields $M_{A s}=M_{B s} \cong 1$ and $Y_{A s}=Y_{B s} \cong Y_{s}$, with $Y_{s}^{2}=$ $Q-1$. Carrying out a linear stability analysis of the rate equations under these conditions (see Appendix B) shows that stable solutions exist under the following conditions.

\section{A. Nonzero detuning}

$$
|\Delta \Omega|<2\left(\eta_{i}+\alpha_{H} \eta_{r}\right),
$$

$$
|\Delta \Omega|>2\left(\eta_{i}+\alpha_{H} \eta_{r}\right) \sqrt{1-\left[\frac{Q}{2 \tau_{N}\left(\eta_{i}-\alpha_{H} \eta_{r}\right)}\right]^{2}} .
$$

These approximate stability conditions fail only in the case of $\alpha_{H}=0, \eta_{i}=0$. To the best of our knowledge these conditions have not been reported previously.

\section{B. Zero detuning}

1. In-phase $\left(\phi_{s}=0\right)$

$$
\begin{gathered}
\eta_{i}+\alpha_{H} \eta_{r}-\frac{2 Q \tau_{p}|\eta|^{2}}{(Q-1)}<0, \\
(Q-1)\left[Q-2 \tau_{N}\left(\eta_{i}-\alpha_{H} \eta_{r}\right)\right] \\
-4 \eta_{i} \tau_{p}\left(Q^{2}-4 \eta_{i} \tau_{N} Q+4 \tau_{N}^{2}|\eta|^{2}\right)>0 .
\end{gathered}
$$

\section{Antiphase $\left(\phi_{s}=\pi\right)$}

$$
\begin{gathered}
\eta_{i}+\alpha_{H} \eta_{r}+\frac{2 Q \tau_{p}|\eta|^{2}}{(Q-1)}<0, \\
(Q-1)\left[Q+2 \tau_{N}\left(\eta_{i}-\alpha_{H} \eta_{r}\right)\right] \\
+4 \eta_{i} \tau_{p}\left(Q^{2}+4 \eta_{i} \tau_{N} Q+4 \tau_{N}^{2}|\eta|^{2}\right)>0 .
\end{gathered}
$$

Equations (32), (33), and (35) are equivalent to Eqs. (10), (11), and (12), respectively, of [14]. For the special case $\eta_{i}=0$, Eqs. (32) and (35) reproduce Eqs. (7) and (6), respectively, of [8]. Since attention is now restricted to the situation where $\left|\tau_{p} \eta_{i}\right| \ll 1$ and $\left|\tau_{p} \eta_{r}\right| \ll 1$, it is worth noting that for most practical cases of interest the important condition from Eqs. (32) and (34) is simply the sign of $\left(\eta_{i}+\alpha_{H} \eta_{r}\right)$. Thus, 
in terms of phase $\theta$ of the coupling coefficient, the stability changes at points where $\tan \theta=-\alpha_{H}$.

\section{DYNAMICS OF TWO LATERALLY COUPLED SEMICONDUCTOR LASERS}

The dynamics of the system described above have been studied by three methods: direct numerical integration of the rate equations, small-signal stability analysis, and study of bifurcations using the standard software package AUTO [29]. The results will be discussed for each of the structures in turn, beginning with the real index guide whose coupling coefficient is illustrated in Fig. 2(a). It should be noted that we choose detuning (expressed in linear frequency $=\Delta \Omega / 2 \pi$ ), laser separation (in the form $d / a$ ), and pump ratio, $P / P_{t h}$, as our primary bifurcation parameters to study the dynamics, and focus only on two basic bifurcations of stationary points [30,31], i.e., saddle-node and Hopf bifurcations, computed with the package AUTO in order to compare with those obtained from the approximate expressions.

\section{A. Real index guide with $\Delta n=0.000971$ with no gain-guiding}

The parameters of this structure are given in the text following Eq. (24) in Sec. III. Figures 3 and 4 show the stability boundaries in terms of detuning versus $d / a$ for the cases of normalized pumping $P / P_{t h}=1.1$ and 2 , respectively, with $\alpha_{H}=2$ for both cases. The solid blue lines (labeled " $\mathrm{S}$ ") denote the saddle-node bifurcations derived from continuation, while the broken green curves correspond to the approximation given by Eq. (30). The solid red lines (labeled "H") denote the calculated Hopf bifurcation while the broken black curves are the approximation given by Eq. (31). We stress that the difference between supercritical and subcritical bifurcations is not indicated in these figures. The reason is twofold. First, all curves in both figures are supercritical bifurcations (of stable solutions) within the considered range of coupling parameters. Second and more importantly, we focus on the level of agreement between continuation and approximation results. From Figs. 3 and 4, we see that the approximations computed from the derived equations, in spite of their apparent

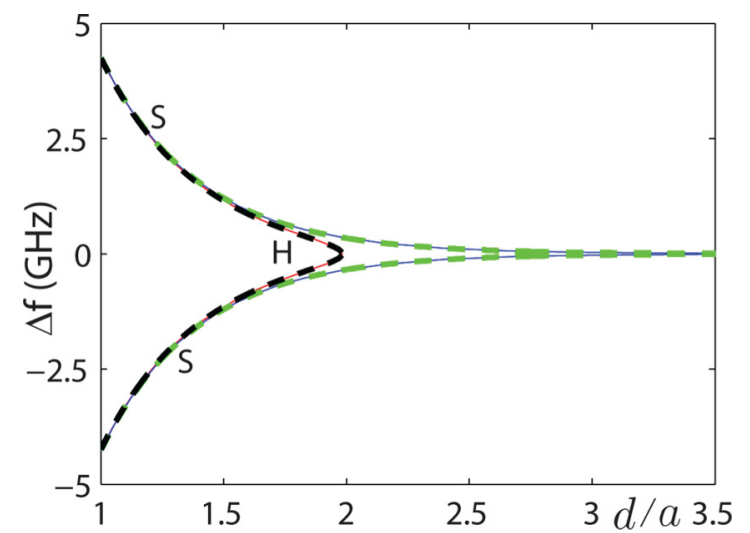

FIG. 3. Stability boundaries in terms of detuning versus $d / a$ for the real index guide in the case of normalized pumping $P / P_{t h}=$ 1.1 and $\alpha_{H}=2$. "H" denotes the Hopf bifurcation; " $\mathrm{S}$ " denotes the saddle-node bifurcation.

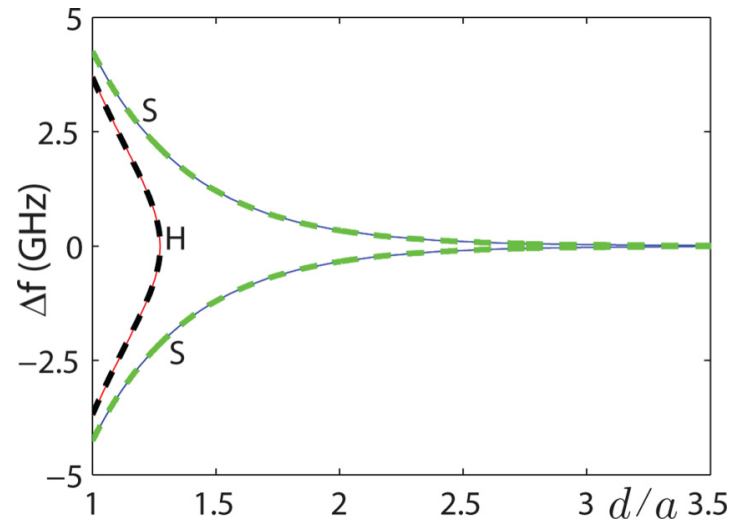

FIG. 4. Stability boundaries in terms of detuning versus $d / a$ for the real index guide in the case of normalized pumping $P / P_{t h}=2$ and $\alpha_{H}=2$. "H" and "S" as in Fig. 3.

simplicity, are in excellent agreement with the accurate results in all cases. The overall shapes of the characteristics are very similar to those presented for the case $\alpha_{H}=2, a=2 \mu \mathrm{m}$ in Ref. [17], Fig. 12(c), using a coupled-laser model.

An alternative approach to detecting various bifurcations is to analyze intensity time series obtained from direct numerical integration of the rate equations [31]. We are now in a position to connect one-parameter bifurcation diagrams obtained by this method with continuation results and approximations. To this end, we compute the bifurcation diagrams in terms of extrema (maxima and minima) of the intensities in laser $\mathrm{A}$ as a function of $d / a$. Two examples of one-parameter bifurcation diagrams are shown in Figs. 5(a) and 5(b) for the case of $\alpha_{H}=$ $2, P / P_{t h}=1.1$, with frequency detunings of 0 and $-1 \mathrm{GHz}$, respectively. It is known that the coupled-laser system admits stable continuous wave $(\mathrm{cw})$ solutions with identical laser amplitude at zero detuning for large enough values of $d / a$ and is destabilized through a supercritical Hopf bifurcation with decreasing $d / a$; for nonzero detuning, a pair of $\mathrm{cw}$ solutions is created via a saddle-node bifurcation and the stable branch bifurcates into a stable periodic oscillation (limit cycle) through a supercritical Hopf bifurcation as $d / a$ is decreased. As can be seen from Fig. 5, the regions of instability in these diagrams correspond to the appropriate regions bounded by the Hopf bifurcations in Fig. 3. Within the instability regions (as we decrease $d / a$ from the Hopf bifurcations), the system

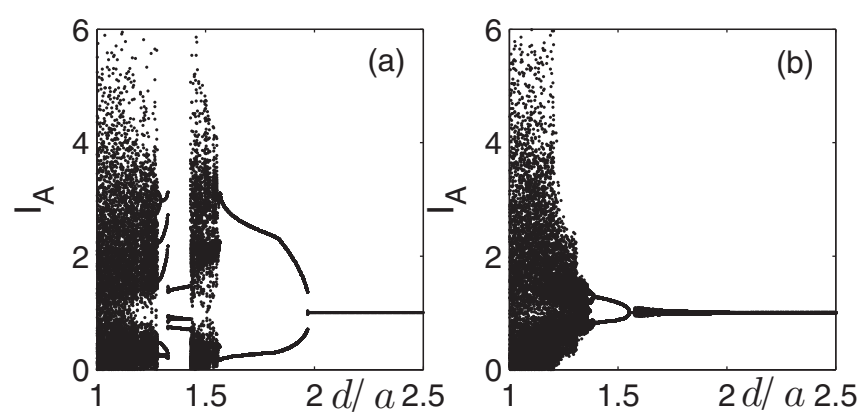

FIG. 5. Bifurcation diagrams for extrema of the intensities in laser A for the real index guide with no gain-guiding in the case of $\alpha_{H}=2$, $P / P_{t h}=1$.1, with frequency detunings of (a) 0 and (b) $-1 \mathrm{GHz}$. 


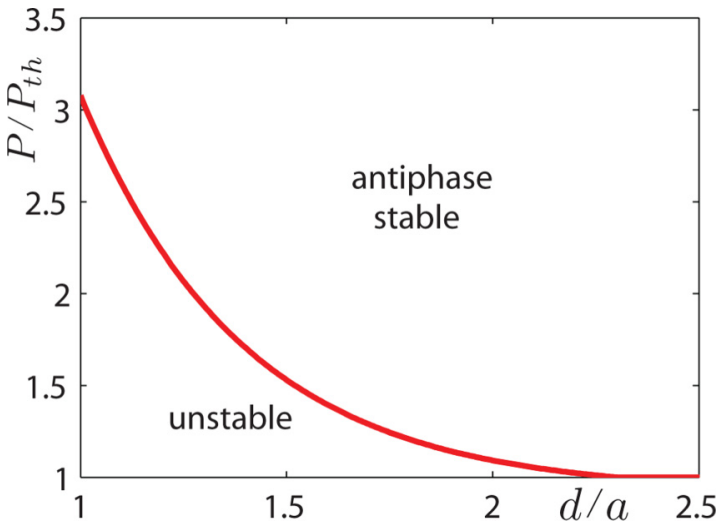

FIG. 6. The stability boundary for zero detuning in the real index guide, as given by Eq. (35) in terms of $P / P_{t h}$ versus $d / a$ for $\alpha_{H}=2$.

undergoes further bifurcations and finally exhibits a more complicated and rich dynamics. Additionally, we note that the overall shapes of the diagrams, with distinct regions of stability (phase-locked cw solutions), periodicity/quasiperiodicity, and chaotic behavior, are similar to that presented for the case $\alpha_{H}=2, a=2 \mu \mathrm{m}$ in Ref. [17], Fig. 6(c3), using a composite-cavity model. This similarity gives confidence when comparing and interpreting the bifurcation results of the other three waveguiding structures.

To further validate our approximations, we revisit the case of zero detuning. For zero detuning the in-phase solutions are always unstable for the considered range of $d / a$, so we show in Fig. 6 only the boundary (Hopf bifurcation) between stable antiphase solutions and instability on a plot of pumping relative to threshold, $P / P_{t h}$, versus $d / a$ for the case of zero detuning with $\alpha_{H}=2$, found from the solution of Eq. (35). Comparison of this boundary curve with the bifurcation diagram in Fig. 5(a) and the two-parameter bifurcation diagrams in Figs. 3 and 4 confirms that the approximation of Eq. (35) provides an accurate guide to the stability for zero detuning. In addition, a comparison among Figs. 3, 4, and 6 shows that the instability region grows in size with decreasing pump level, which will also be seen in the other three waveguiding structures.

\section{B. Real index guide with $\Delta \boldsymbol{\Delta}=\mathbf{0 . 0 0 0 5}$ and gain-guiding}

This structure has the parameters given in the first line of Table I, and the variation of the corresponding real and imaginary parts of the coupling coefficient are illustrated in Fig. 2(b). The numerical continuation results in terms of detuning versus $d / a$ for the case of normalized pumping $P / P_{t h}=1.1$ with $\alpha_{H}=2$ are shown in Fig. 7. Here the bold blue lines denote the supercritical saddle-node bifurcations and the bold red curve denotes the supercritical Hopf bifurcation, while the thin red line refers to the subcritical Hopf bifurcation. The supercritical bifurcation curves can be compared directly with the results obtained from direct numerical integration, while the subcritical one is presented to achieve a consistent bifurcation diagram [31]. In contrast to the case of purely real index guiding $(\Delta n=0.000971)$, there are two separate Hopf branches within the range of $d / a$ considered in the case here of a real index guide with gain-guiding; the upper and the lower saddle-node branches cross each other around $d / a=2.5$

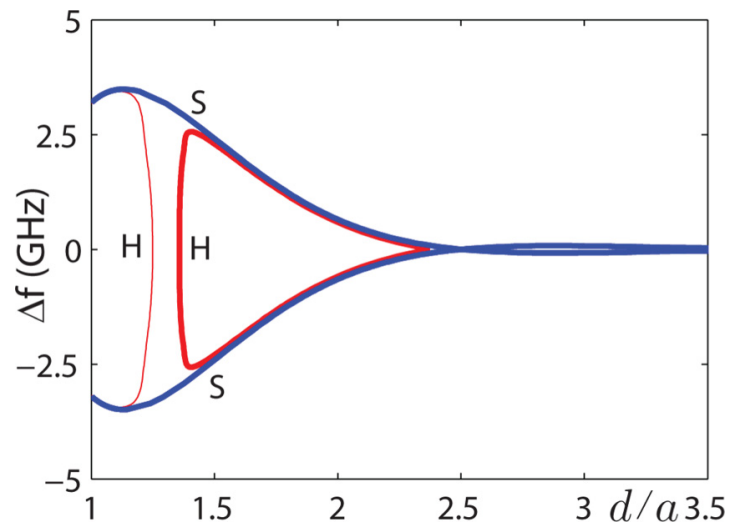

FIG. 7. Stability boundaries in terms of detuning versus $d / a$ for the structure with parameters given in the first line of Table I in the case of normalized pumping $P / P_{t h}=1.1$ and $\alpha_{H}=2$. Bold lines correspond to the supercritical bifurcations and the thin line to the subcritical case. "H" and "S" as in Fig. 3.

(more precisely, there are two opposite codimension-2 cusp bifurcations forming the intersection, which is not visible at the current scale), resembling the onset of a bifurcation cascade behavior. This indicates that the coupling coefficient plays an important role in determining the underlying dynamics of the two coupled lasers. In Fig. 8 these results are compared with the approximations of Eqs. (30) and (31), using the same definition of the curves as used for Figs. 3 and 4. The level of agreement between the approximations and the exact results is very good, demonstrating that Eqs. (30) and (31) are simple, yet powerful, tools in assessing the stability boundaries for this system.

As in the preceding case of purely real index guiding, we compare the continuation results with those calculated by numerically integrating the rate equations to help us understand the bifurcation details. Hence we show in Figs. 9(a) and 9(b) one-parameter bifurcation diagrams, shown as extrema of the intensities in laser A, for the case of $\alpha_{H}=2, P / P_{t h}=1.1$, with frequency detunings of 0 and $-1 \mathrm{GHz}$, respectively. As expected, the regions of instability in these diagrams correspond to the appropriate regions bounded by two Hopf

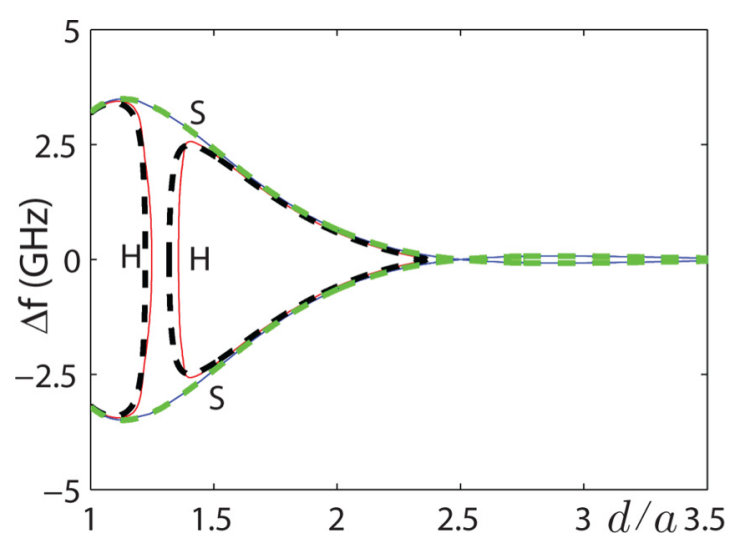

FIG. 8. Stability boundaries as in Fig. 7, together with the corresponding approximate results from Eqs. (30) and (31). Line types and color code as for Figs. 3 and 4. "H" and "S" as in Fig. 3. 

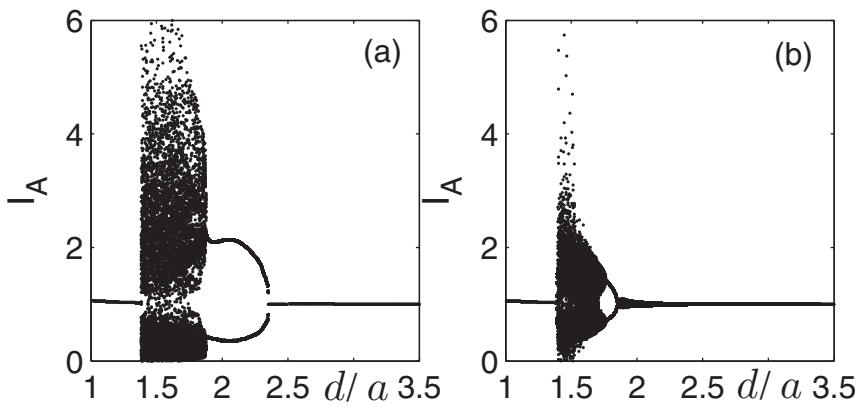

FIG. 9. Bifurcation diagrams for extrema of the intensities in laser A for the structure with parameters given in the first line of Table I in the case of $\alpha_{H}=2, P / P_{t h}=1.1$, with frequency detunings of (a) 0 and (b) $-1 \mathrm{GHz}$.

bifurcations, which are completely determined by the closed supercritical Hopf branch in Fig. 7.

To further test our approximations, we consider the stability boundary for zero detuning in the plane of pumping relative to threshold, $P / P_{t h}$, versus $d / a$. Unlike the previous structure, the system dynamics in the structure with parameters given in the first line of Table I are totally determined by the stability of in-phase solutions in the $d / a$ interval considered. Figure 10 shows the boundary between stable in-phase solutions and instability for $\alpha_{H}=2$, found from the solution of Eqs. (32) and (33). In this figure, the blue curve stands for the Hopf branch and the straight black line represents the pitchfork branch: on the left side of the black line, the laser dynamics is determined by the in-phase solutions (note that the antiphase solutions are unstable everywhere), whereas on the right side, the system admits stable antiphase solutions in the range of $d / a$ shown. It is worth noting that in the case of nonzero detuning, instead of pitchfork bifurcations saddle-node ones become the boundary. In particular, even vanishingly small detuning can destroy the symmetries in our system and thus the pitchfork bifurcations unfold to saddle-node bifurcations, which is common for symmetrically coupled systems $[27,32,33]$. Comparison of this boundary curve with the bifurcation diagram in Fig. 9(a) and the two-parameter bifurcation diagrams in Figs. 7

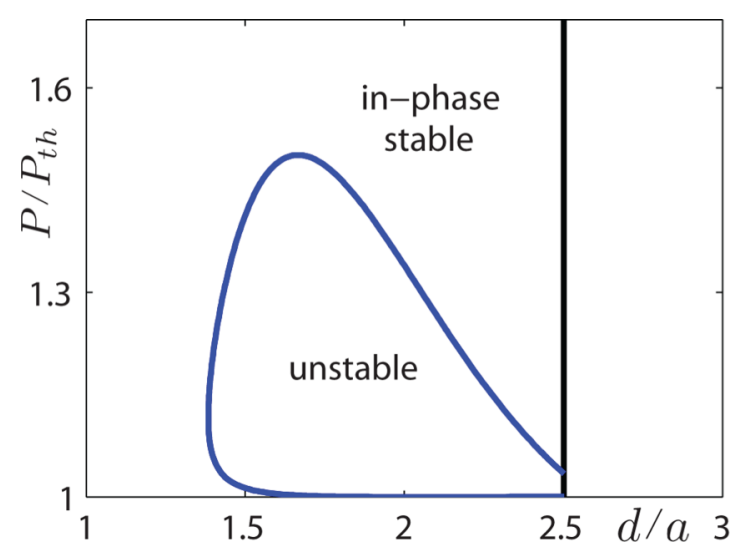

FIG. 10. The stability boundary for zero detuning in the structure with parameters given in the first line of Table I, as given by Eqs. (32) and (33) in terms of $P / P_{t h}$ versus $d / a$ for $\alpha_{H}=2$.

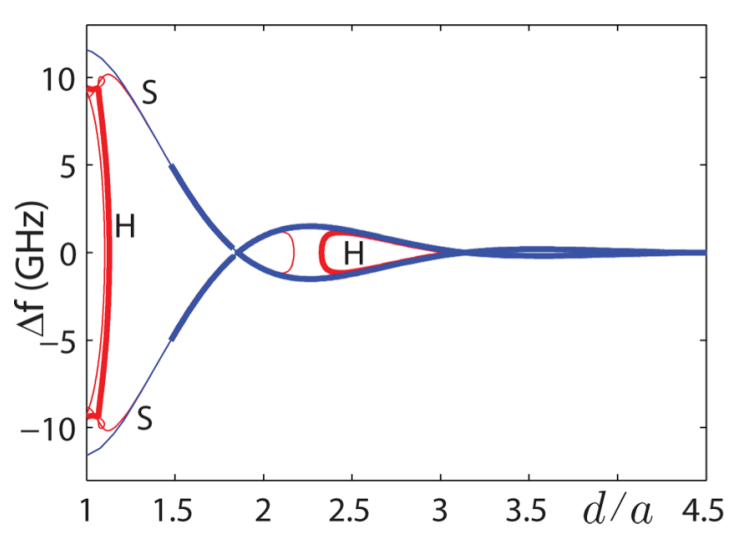

FIG. 11. Stability boundaries in terms of detuning versus $d / a$ for the structure with parameters given in the second line of Table I in the case of normalized pumping $P / P_{t h}=1.1$ and $\alpha_{H}=2$. Bold lines correspond to the supercritical bifurcations and thin lines to the subcritical cases. "H" and "S" as in Fig. 3.

and 8 confirms that the approximation of Eqs. (32) and (33) provides an accurate guide to the stability for zero detuning.

\section{Guide with $\Delta \boldsymbol{n}=\mathbf{0}$, pure gain-guiding}

This structure has the parameters given in the second line of Table I, and the variation of the corresponding real and imaginary parts of the coupling coefficient are illustrated in Fig. 2(c). The bifurcations in terms of detuning versus $d / a$ for the case of normalized pumping $P / P_{t h}=1.1$ with $\alpha_{H}=2$ are shown in Fig. 11, with the same convention for line types and colors as used in Fig. 7. As expected, we observe that the periodic behavior of the real and imaginary parts of the coupling coefficient, noted in Sec. III, has a strong effect on the dynamics as described by these bifurcations. In this two-parameter bifurcation diagram, the two saddle-node branches cross each other twice and more Hopf branches appear. This suggests that a bifurcation cascade between apparently stable and unstable regions should be observed in the bifurcation diagram obtained from direct numerical integration. Interestingly, it is clearly seen that the bifurcation diagram consists of more segments of supercritical and subcritical bifurcations compared with the preceding case. This is due to the appearance of several codimension-2 saddle-node-Hopf points (not shown) where saddle-node and Hopf curves are tangent and they change from supercritical to subcritical [31]. Besides, these codimension-2 points are known as origins of complex bifurcation structures giving rise to chaos. Apart from saddle-node-Hopf points, there are several other codimension-2 bifurcation points, such as the socalled generalized Hopf bifurcation and cusp bifurcation [34]. However, finding secondary bifurcation curves and other codimension-2 bifurcation points is outside the scope of the current paper. Figure 12 illustrates the bifurcation diagram in the plane of detuning and $d / a$ obtained both from continuation and from the approximations of Eqs. (30) and (31), using the same definition of the curves as used previously. The level of agreement between the approximations and the exact results is generally very good, although in the case of the two parallel Hopf bifurcation curves that are very close to each other for 


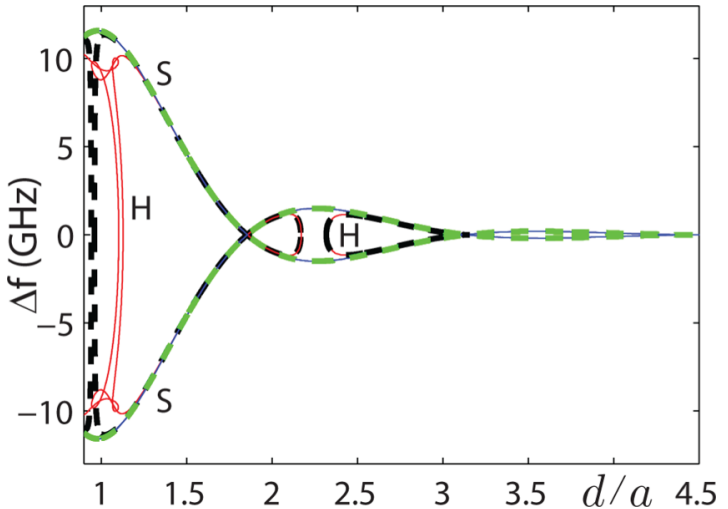

FIG. 12. Stability boundaries as in Fig. 11, together with the corresponding approximate results from Eqs. (30) and (31). Line types and color code as for Figs. 3 and 4. "H" and "S" as in Fig. 3.

values of $d / a$ just above 1.0, the approximation overestimates the corresponding coupling strengths, i.e., predicting two Hopf curves for values of $d / a$ slightly below 1.0. Quantitative discrepancies between continuation results and our approximations for the absolute position of the two Hopf bifurcation lines are probably related to the neglected higher order terms as well as other simplifications made in Appendix B.

Our small-signal analysis of the rate equations indicates that stability boundaries may be associated with two important physical parameters, namely, the linewidth enhancement parameter $\alpha_{H}$ and the pumping ratio $P / P_{t h}$; see Eqs. (30) and (31). Hence it is of particular interest here to determine how the dynamics and the level of agreement between continuation and approximation results vary in terms of these two parameters and how robust the agreement is. A typical example of the effect of changing linewidth enhancement parameter and pumping rate on the dynamics is illustrated in Fig. 13, with the same line type and color convention as that used in Fig. 12. Figure 13(a) shows the bifurcations for the case of $\alpha_{H}=0, P / P_{t h}=1.1$, while Fig. 13(b) is for $\alpha_{H}=2$, $P / P_{t h}=2$. On the one hand, we find that the Hopf bifurcation branches become simpler and Hopf and saddle-node curves overlap significantly for $d / a$ less than about 2.3 due to the choice of vanishingly small linewidth enhancement factor $\alpha_{H}$. On the other hand, compared to Fig. 12, we see that the Hopf branches in Fig. 13(b) shrink towards a narrower range of

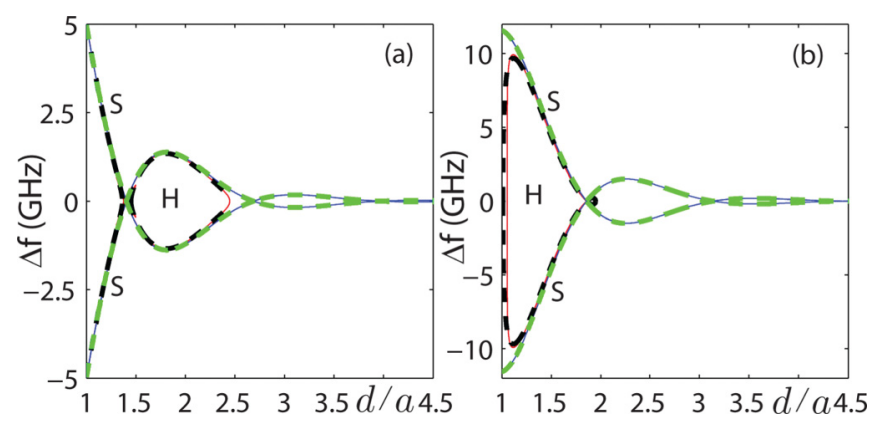

FIG. 13. Stability boundaries for the cases (a) $\alpha_{H}=0, P / P_{t h}=$ 1.1 , (b) $\alpha_{H}=2, P / P_{t h}=2$. The line type and color convention is the same as that used in Fig. 12. "H" and "S" as in Fig. 3.

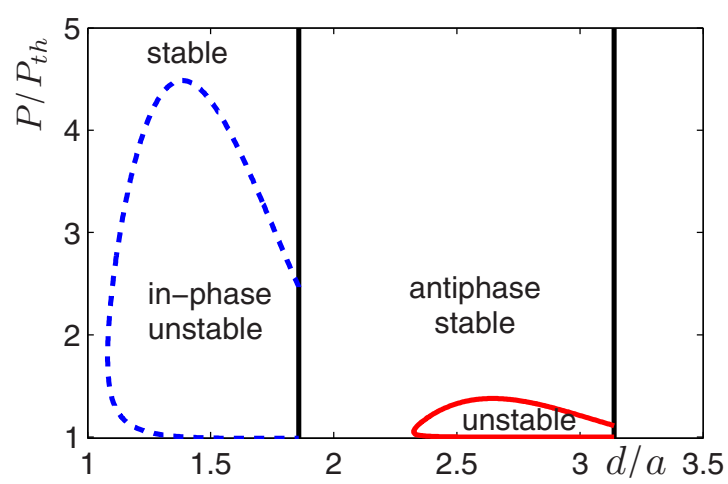

FIG. 14. The stability boundary for zero detuning in the structure with parameters given in the second line of Table I, as given by Eqs. (32)-(35) in terms of $P / P_{t h}$ versus $d / a$ for $\alpha_{H}=2$.

$d / a$, say, between around 1 and 2 . This is due to the fact that Hopf bifurcations are responsible for the instability and larger coupling strengths (smaller values of $d / a$ ) are required to destabilize the coupled laser system for higher pump rates. More importantly, the periodic behavior holds and again the agreement between approximate and exact results is generally very good with changing $\alpha_{H}$ and/or $P / P_{t h}$.

Figure 14 shows the boundary between stable inphase/antiphase solutions and instability on a plot of pumping relative to threshold, $P / P_{t h}$, versus $d / a$ for the case of zero detuning with $\alpha_{H}=2$, found from the solution of Eqs. (32)-(35). The bifurcation diagram is divided into two main regions. In the first part, the system first admits stable in-phase solutions and then can be destabilized at a Hopf bifurcation (broken blue line); in the second part, the system turns to admit stable antiphase solutions and its instability is determined by the Hopf bifurcations (solid red line) occurring on antiphase solutions in an unstable region of much smaller size. Between the in-phase and antiphase regions there is a pitchfork bifurcation boundary (black line) that alters the role of both solutions. Such a bifurcation cascade is expected and, more precisely, the periodic nature of the system is clear here, with regions of in-phase and antiphase stability allowed, depending, to a good degree of accuracy, on the sign of $\left(\eta_{i}+\right.$ $\left.\alpha_{H} \eta_{r}\right)$, as noted above in Sec. IV. The boundaries of the regions of instability are given by the solutions of Eqs. (32) and (33) for the in-phase case (blue/black lines in Fig. 14), and Eqs. (34) and (35) for the antiphase case (red/black lines in Fig. 14).

One-parameter bifurcation diagrams (not shown here) confirm the accuracy of the results in Fig. 14 for the boundaries of instability for zero detuning. Similarly, for finite detunings, calculated one-parameter bifurcation diagrams confirm the accuracy of the boundaries shown in Figs. 11-13 for this case of a structure with pure gain guiding.

\section{Real index antiguide with $\Delta n=-\mathbf{0 . 0 0 0 5}$ and gain-guiding}

This structure has the parameters given in the third line of Table I, and the variation of the corresponding real and imaginary parts of the coupling coefficient are illustrated in Fig. 2(d). The bifurcations in terms of detuning versus $d / a$ for the case of normalized pumping $P / P_{t h}=1.1$ with $\alpha_{H}=2$ are shown in Fig. 15. Periodic behavior is seen over a wider range 


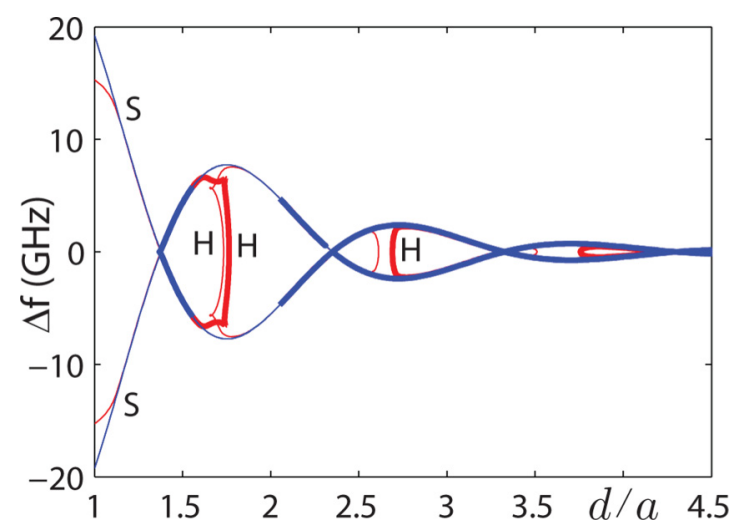

FIG. 15. Stability boundaries in terms of detuning versus $d / a$ for the structure with parameters given in the third line of Table I in the case of normalized pumping $P / P_{t h}=1.1$ and $\alpha_{H}=2$. Bold lines correspond to the supercritical bifurcations and thin lines to the subcritical cases. "H" and "S" as in Fig. 3.

of $d / a$ in this structure than in the others considered. This is due to the reduced damping of the oscillations in the coupling coefficient, as seen in Fig. 2(d), caused by a lower value of $W_{r}$ (see Table I). Additionally, there are even more segments of supercritical and subcritical bifurcations in the bifurcation diagram since, among many others, more codimension-2 saddle-node-Hopf points (not shown) accounting for these stable and unstable changes of the tangent saddle-node and Hopf bifurcation lines appear compared to the case revealed in Fig. 11. In Fig. 16 these results are compared with the approximations of Eqs. (30) and (31), using the same definition of the curves as used previously. The level of agreement between the approximations and the exact results is generally good as expected, although the approximation fails to reproduce the fine details of the bifurcations in the region of $d / a$ near 1.7 , probably due to the same reason given for Fig. 12.

Once again the effect of changing linewidth enhancement parameter and pumping rate is illustrated here in Fig. 17, with good agreement between exact and approximate results. The results also confirm the observations about dynamics in Fig. 13; that is, Hopf and saddle-node curves overlap

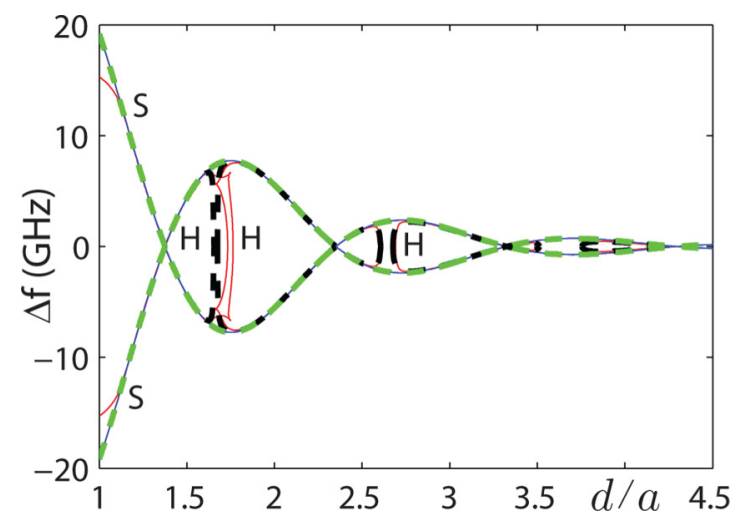

FIG. 16. Stability boundaries as in Fig. 15, together with the corresponding approximate results from Eqs. (30) and (31). Line types and color code as for Figs. 3 and 4. "H" and "S" as in Fig. 3.
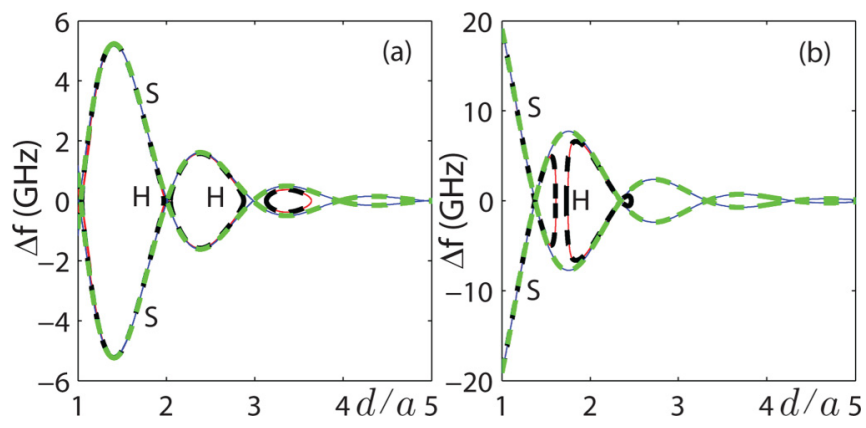

FIG. 17. Stability boundaries for the cases (a) $\alpha_{H}=0, P / P_{t h}=$ 1.1, (b) $\alpha_{H}=2, P / P_{t h}=2$. The line type and color convention is the same as that used in Fig. 16. "H" and "S" as in Fig. 3.

significantly for $\alpha_{H}=0$ and Hopf branches shrink to a narrower interval of small enough laser separation $d / a$ with increasing pump rates.

Figure 18 shows the boundary between stable inphase/antiphase solutions and instability on a plot of pumping relative to threshold, $P / P_{t h}$, versus $d / a$ for the case of zero detuning with $\alpha_{H}=2$, found from the solution of Eqs. (32)-(35). Once again, one-parameter bifurcation diagrams (not shown here) confirm the accuracy of the results in Fig. 18 for the boundaries of instability for zero detuning. The bifurcation diagram is divided into three main regions: in the middle part, the dynamics is dominated by in-phase solutions, while in the left and right parts antiphase solutions switch on and determine the dynamical states of the system; in between them, pitchfork bifurcations take place and switch the onset of in-phase and antiphase solutions each time. Such an interesting bifurcation cascade confirms the periodic behavior seen over a wider range of $d / a$ and determined by the variation of the coupling coefficient in Fig. 2(d). Similarly, for finite detunings, calculated one-parameter bifurcation diagrams confirm the accuracy of the boundaries shown in Figs. 15- 17 for this case of a structure with real index antiguiding and gain-guiding.

\section{CONCLUSION}

The dynamics in two laterally coupled semiconductor lasers have been studied including the effects of gain-guiding and

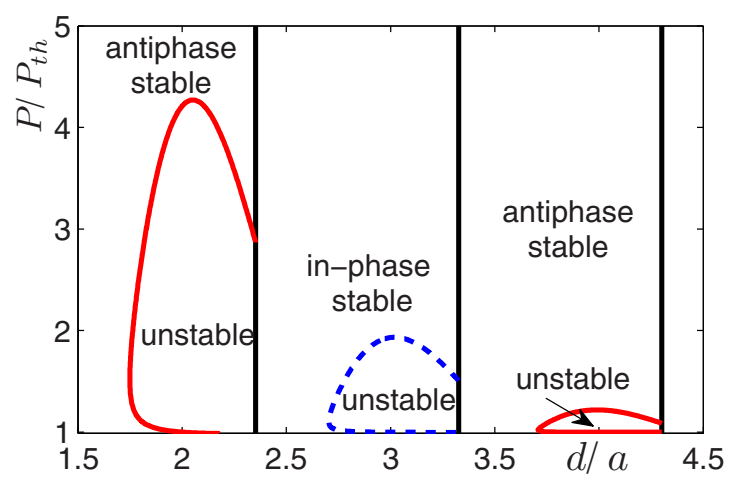

FIG. 18. The stability boundary for zero detuning in the structure with parameters given in the third line of Table I, as given by Eqs. (32)-(35) in terms of $P / P_{t h}$ versus $d / a$ for $\alpha_{H}=2$. 
carrier-induced index antiguiding via a complex coupling coefficient as well as the effects of detuning between the cavity resonances. This approach generalizes previous work where attention is usually confined to real index guides and in most cases the detuning is taken as zero. The work reveals a periodic behavior of the real and imaginary parts of the complex coupling coefficient as the lateral spacing between the lasers is varied. In previous treatments this behavior has been overlooked since for real index guides the damping of the periodicity is large enough to hide the periodicity.

The dynamics of the system described above have been studied primarily by a continuation study of bifurcations using the standard software package AUTO. In addition, however, a small-signal analysis of the rate equations has been carried out using the Routh-Hurwitz stability criteria. For the zero detuning case this analysis reproduces previously published results, but for finite detuning we find analytic results that provide simple expressions for the bifurcations. Comparison of the small-signal results with those from AUTO for variation of laser spacing, pumping, and the linewidth enhancement factor show very good agreement in general. Direct numerical integration of the rate equations provides further support for the accuracy of the predicted boundaries of stable and unstable behavior.

The three methods outlined above, namely, AUTO, smallsignal analysis, and numerical integration, have been applied to four specific examples of coupled lasers with different guiding structures. These are a real index guide, an index guide with gain-guiding, a structure with pure gain-guiding, and a real index antiguide with gain-guiding. Realistic laser parameters for these structures have been chosen in such a way as to ensure that each individual laser would only have a single transverse mode if operated alone. It is found that, following the sequence of structures as defined above, the periodic behavior with laser spacing increases from the pure index guide through to the index antiguide with gain-guiding. This periodicity is exhibited in the development of the bifurcations through the sequence of structures, for both cases of zero and nonzero detuning between the lasers. The general trends found in this comparative study of laser guiding structures are applicable to a range of EELs and VCSELs used in laser arrays, which could have important implications for their design for specific applications. For example, tuning of coupling strength in photonic crystal microcavities can lead to modal switching between in-phase and antiphase modes [35], and detuning of the resonant wavelengths by differential current injection in VCSEL arrays is a fast and efficient method of beam steering [36]. Another example is arrays for high-power sources. This would require further analysis of specific laser arrays in terms of how the dynamics depends on operating parameters and design. Such an analysis will be the subject of future work.

\section{ACKNOWLEDGMENTS}

This work was supported by the Engineering and Physical Sciences Research Council (Grant No. EP/M024237/1). N.L. is grateful to J. Sieber (University of Exeter) for fruitful discussions of bifurcation analysis using the continuation software package AUTO.

\section{APPENDIX A: DERIVATION OF COUPLED-MODE RATE EQUATIONS}

We start from the wave equation for the electric field $\mathbf{E}$ and polarization $\mathbf{P}$ :

$$
\nabla^{2} \mathbf{E}=\frac{\varepsilon_{j}}{c^{2}} \frac{d^{2} \mathbf{E}}{d t^{2}}+\mu_{0} \sigma \frac{d \mathbf{E}}{d t}+\mu_{0} \frac{d^{2} \mathbf{P}}{d t^{2}},
$$

where $\mu_{0}$ is the magnetic permeability, $\sigma$ is the conductivity, $\varepsilon_{j}$ is the dielectric permittivity, and $c$ is the speed of light. If $z$ is the direction of propagation and $x$ is the direction of confinement for a two-dimensional waveguide, then $\mathbf{E}$ and $\mathbf{P}$ are complex functions of $z, x$, and time $t$, and $\varepsilon_{j}$ is a complex function of $x$. Writing these in terms of amplitude and phase, and separating the spatial and temporal components for two coupled waveguides, gives the forms

$$
\begin{aligned}
\mathbf{E}= & \Psi_{A}(x, z)\left|E_{A}(t)\right| \exp \left[-i \phi_{A}(t)-i \omega t\right] \\
& +\Psi_{B}(x, z)\left|E_{B}(t)\right| \exp \left[-i \phi_{B}(t)-i \omega t\right], \\
\mathbf{P}= & \Psi_{A}(x, z) P_{A}(t) \exp \left[-i \phi_{A}(t)-i \omega t\right] \\
& +\Psi_{B}(x, z) P_{B}(t) \exp \left[-i \phi_{B}(t)-i \omega t\right],
\end{aligned}
$$

where $E_{A}=\left|E_{A}\right| e^{-i \phi_{A}}$ and $E_{B}=\left|E_{B}\right| e^{-i \phi_{B}}$. The slowly varying approximation means that the terms $\ddot{E}_{A}, \ddot{E}_{B}, \ddot{P}_{A}, \ddot{P}_{B}$, $\left|\dot{E}_{A}\right| \dot{\phi}_{A},\left|E_{B}\right| \dot{\phi}_{B}, \sigma \dot{E}_{A}, \sigma \dot{E}_{B}, \sigma \dot{\phi}_{A}, \sigma \dot{\phi}_{B}, \dot{\phi}_{A} \dot{P}_{A}, \dot{\phi}_{B} \dot{P}_{B}, \dot{\phi}_{A} P_{A}$, $\dot{\phi}_{B} P_{B}, \dot{P}_{A}, \dot{P}_{B}, \ddot{\phi}_{A}$, and $\ddot{\phi}_{B}$ can be ignored in what follows. Hence

$$
\begin{gathered}
\frac{d^{2} \mathbf{E}}{d t^{2}}=-\Psi_{A}(x, z)\left(\omega^{2} E_{A}+i 2 \omega \frac{d E_{A}}{d t}\right) \exp [-i \omega t] \\
-\Psi_{B}(x, z)\left(\omega^{2} E_{B}+i 2 \omega \frac{d E_{B}}{d t}\right) \exp [-i \omega t] \\
\sigma \frac{d \mathbf{E}}{d t}=-i \sigma \omega \Psi_{A}(x, z) E_{A} \exp [-i \omega t] \\
\frac{d^{2} \mathbf{P}}{d t^{2}}=-i \sigma \omega \Psi_{B}(x, z) E_{B} \exp [-i \omega t] \\
-\omega^{2} \Psi_{A}(x, z) P_{A} \exp [-i \omega t] \\
{ }^{2} \Psi_{B}(x, z) P_{B} \exp [-i \omega t]
\end{gathered}
$$

From Eq. (A2) it follows that

$$
\begin{aligned}
\nabla^{2} \mathbf{E}= & \nabla^{2} \Psi_{A}(x, z) E_{A}(t) \exp [-i \omega t] \\
& +\nabla^{2} \Psi_{B}(x, z) E_{B}(t) \exp [-i \omega t] .
\end{aligned}
$$

Substituting Eqs. (A4)-(A7) in Eq. (A1), and omitting the arguments $x, z, t$ for compactness, gives

$$
\begin{aligned}
& {\left[\nabla^{2} \Psi_{A}+\frac{\omega^{2} \varepsilon_{j}}{c^{2}} \Psi_{A}\right] E_{A}} \\
& +\left[\nabla^{2} \Psi_{B}+\frac{\omega^{2} \varepsilon_{j}}{c^{2}} \Psi_{B}\right] E_{B}+2 i \omega \frac{\varepsilon_{j}}{c^{2}}\left[\frac{d E_{A}}{d t} \Psi_{A}+\frac{d E_{B}}{d t} \Psi_{B}\right] \\
& +i \mu_{0} \sigma \omega\left(\Psi_{A} E_{A}+\Psi_{B} E_{B}\right)+\mu_{0} \omega^{2}\left(\Psi_{A} P_{A}+\Psi_{B} P_{B}\right)=0 .
\end{aligned}
$$

The waveguide configuration means that $\varepsilon_{j}=\varepsilon_{1}$ in the waveguide cores and $\varepsilon_{j}=\varepsilon_{2}$ for all other values of $x$. 
For guide A, in the absence of guide $\mathrm{B}, E=\Psi_{A}(x, z)$ satisfies the wave equation

$$
\nabla^{2} \Psi_{A}+\varepsilon_{A} k_{A}^{2} \Psi_{A}=0,
$$

where $\varepsilon_{A}=\varepsilon_{1}$ for $|x|<a$, and $\varepsilon_{A}=\varepsilon_{2}$ for $a<|x|$.

For guide $\mathrm{B}$, in the absence of guide A, $E=\Psi_{B}(x, z)$ satisfies the wave equation

$$
\nabla^{2} \Psi_{B}+\varepsilon_{B} k_{B}^{2} \Psi_{B}=0,
$$

where $\varepsilon_{B}=\varepsilon_{1}$ for $-3 a-2 d<x<-a-2 d$, and $\varepsilon_{B}=\varepsilon_{2}$ for $x<-3 a-2 d$ or $-a-2 d<x$.

Using Eqs. (A9) and (A10) in Eq. (A8) yields

$$
\begin{aligned}
\frac{d E_{A}}{d t} \Psi_{A}+\frac{d E_{B}}{d t} \Psi_{B}= & \frac{i}{2 \omega \epsilon_{j}}\left[\left(\omega^{2} \varepsilon_{j}-\Omega_{A}^{2} \varepsilon_{A}\right) \Psi_{A} E_{A}\right. \\
& \left.+\left(\omega^{2} \varepsilon_{j}-\Omega^{2} \varepsilon_{B}\right) \Psi_{B} E_{B}\right] \\
& -\frac{\mu_{0} c^{2} \sigma}{2 \varepsilon_{j}}\left(\Psi_{A} E_{A}+\Psi_{B} E_{B}\right) \\
& +\frac{i \mu_{0} c^{2} \omega}{2 \varepsilon_{j}}\left(\Psi_{A} P_{A}+\Psi_{B} P_{B}\right),
\end{aligned}
$$

where $\Omega_{A, B}=c k_{A, B}$.

Multiplying Eq. (A11) by $\Psi_{A}^{*}$ and integrating over $x$ gives

$$
\begin{aligned}
& \frac{d E_{A}}{d t} \int\left|\Psi_{A}\right|^{2} d x \\
& =\frac{i}{2 \omega \varepsilon_{j}}\left[E_{A} \int \Psi_{A}^{*}\left(\omega^{2} \varepsilon_{j}-\Omega_{A}^{2} \varepsilon_{A}\right) \Psi_{A} d x\right. \\
& \left.\quad+E_{B} \int \Psi_{A}^{*}\left(\omega^{2} \varepsilon_{j}-\Omega_{B}^{2} \varepsilon_{B}\right) \Psi_{B} d x\right] \\
& \quad-\frac{\mu_{0} c^{2} \sigma}{2 \varepsilon_{j}} E_{A} \int\left|\Psi_{A}\right|^{2} d x+\frac{i \mu_{0} c^{2} \omega}{2 \varepsilon_{j}} \int\left|\Psi_{A}\right|^{2} P_{A} d x,
\end{aligned}
$$

where it has been assumed that overlap terms $\int \Psi_{A}^{*} \Psi_{B} d x$ and $\int \Psi_{A}^{*} \Psi_{B} P_{B} d x$ are negligible, and the limits on the integrals are from $-\infty$ to $\infty$. A similar equation is found for $\Psi_{B}$.

In practice, the values of detuning $\left(\Omega_{A}-\Omega_{B}\right)$ are much smaller than the frequencies $\Omega_{A, B}$, and it therefore follows that the difference of wave numbers $\left(k_{A}-k_{B}\right)$ is much smaller than the values of $k_{A, B}$. Hence, in evaluating the integrals for Eq. (A12) it is safe to neglect these differences and assume $k_{A}=k_{B} \equiv k, U_{A}=U_{B} \equiv U, W_{A}=W_{B} \equiv W$, and $V_{A}=V_{B} \equiv V$ in the expressions for $\Psi_{A}$ and $\Psi_{B}$. However, the difference between $\omega$ and $\Omega_{A}$ must be retained in the integrand $\left(\omega^{2} \varepsilon_{j}-\Omega_{A}^{2} \varepsilon_{A}\right)$ of the self-coupling term in Eq. (A12). A further simplification is possible in this term, since $\left(\omega^{2}-\Omega_{A}^{2}\right) \approx 2 \omega\left(\omega-\Omega_{A}\right)$.

The term in conductivity in Eq. (A12) can be interpreted as the total cavity losses (end losses) and written in terms of the cavity loss rate $\gamma_{E}$ :

$$
\frac{\mu_{0} c^{2} \sigma}{2 n_{j}^{2}}=\frac{\gamma_{E}}{2}
$$

The term in polarization $P_{A}$ in Eq. (A12) can be expressed in terms of the gain $g_{A}$ and index change $\delta n_{1}$ in the core of waveguide $\mathrm{A}$, and in terms of the loss $\alpha$ in the cladding regions:

$$
\begin{aligned}
& \frac{i \mu_{0} c^{2} \omega}{2 \varepsilon_{j}} \int\left|\Psi_{A}\right|^{2} P_{A} d x \\
& =\frac{1}{2}\left[\frac{c}{n_{g}}\left(g_{A}-\alpha\right)+i \frac{\omega}{n_{g}} \delta n_{1}\right] E_{A} \int_{-a}^{a}\left|\Psi_{A}\right|^{2} d x \\
& \quad-\frac{c}{2 n_{g}} \alpha E_{A}\left[\int_{-\infty}^{-a}\left|\Psi_{A}\right|^{2} d x+\int_{a}^{\infty}\left|\Psi_{A}\right|^{2} d x\right],
\end{aligned}
$$

where $g_{A}-g_{\text {Ath }}=a_{\text {diff }}\left(N_{A}-N_{A t h}\right)$ and $\delta n_{1}=-(c / \omega) a_{\text {diff }}$ $\alpha_{H}\left(N_{A}-N_{A t h}\right)$, with $N_{A}$ as the carrier concentration, $a_{\text {diff }}$ as the differential gain, $\alpha_{H}$ as the linewidth enhancement factor, $n_{g}$ as the group index, and the subscript " $t h$ " indicating the threshold values. Here we have assumed that there is a constant concentration of carriers in the core region of each waveguide.

Substituting the forms for the even-order fields from Eq. (4) and including Eqs. (A13) and (A14), Eq. (A12) can be written as

$$
\begin{aligned}
\frac{d E_{A}}{d t} I_{2}= & \frac{i}{2 \omega n^{2}}\left[E_{A} \varepsilon_{1} 2 \omega\left(\omega-\Omega_{A}\right) I_{2}\right. \\
& \left.+E_{B} \omega^{2}\left(\varepsilon_{1}-\varepsilon_{2}\right) I_{3} \exp \left(-2 W \frac{d}{a}\right)\right] \\
& -\frac{\gamma_{E}}{2} E_{A} I_{2}+\frac{c}{2 n_{g}}\left[g_{A t h}+a_{\mathrm{diff}}\left(N_{A}-N_{A t h}\right)\right. \\
& \left.\times\left(1-i \alpha_{H}\right)\right] E_{A} I_{1}-\frac{c}{2 n_{g}} \alpha E_{A} I_{2},
\end{aligned}
$$

where

$$
\begin{aligned}
& I_{1}=2 \int_{0}^{a}\left|\cos \left(U \frac{x}{a}\right)\right|^{2} d x, \\
& I_{2}=I_{1}+2|\cos (U)|^{2} e^{2 W_{r}} \int_{a}^{\infty} e^{-2 W_{r} x / a} d x, \\
& I_{3}=\cos (U) e^{-W} \int_{-a}^{a} \cos \left(U^{*} \frac{x}{a}\right) e^{-W x / a} d x .
\end{aligned}
$$

Equation (A15) can be written in the more conventional form

$$
\begin{aligned}
\frac{d E_{A}}{d t}= & \Gamma \frac{c}{n_{g}}\left[g_{A t h}+a_{\mathrm{diff}}\left(N_{A}-N_{A t h}\right)\left(1-i \alpha_{H}\right)\right] E_{A} \\
& -\frac{E_{A}}{2 \tau_{p}}+i\left(\omega-\Omega_{A}\right) E_{A}+i \eta E_{B},
\end{aligned}
$$

where $\Gamma$ is the optical confinement factor defined as $\Gamma=I_{1} / I_{2}$ and all losses (absorption, scattering, and end loss) have been combined into a photon lifetime $\tau_{p}$, defined as $1 / \tau_{p}=\gamma_{E}+$ $\alpha c / n_{g}$. The coupling coefficient $\eta$ is given by

$$
\eta=\frac{c k}{n}\left(\Delta n+i \frac{g}{2 k}\right) \frac{I_{3}}{I_{2}} \exp \left(-2 W \frac{d}{a}\right) .
$$

Equation (A17) can be simplified with the aid of the threshold condition Eq. (12) for the solitary laser:

$$
\begin{aligned}
\frac{d E_{A}}{d t}= & \Gamma \frac{c}{2 n_{g}} a_{\mathrm{diff}}\left(N_{A}-N_{A t h}\right)\left(1-i \alpha_{H}\right) E_{A} \\
& +i\left(\omega-\Omega_{A}\right) E_{A}+i \eta E_{B} .
\end{aligned}
$$


The corresponding rate equation for the field in waveguide $\mathrm{B}$ is given by

$$
\begin{aligned}
\frac{d E_{B}}{d t}= & \Gamma \frac{c}{2 n_{g}} a_{\mathrm{diff}}\left(N_{B}-N_{B t h}\right)\left(1-i \alpha_{H}\right) E_{B} \\
& +i\left(\omega-\Omega_{B}\right) E_{B}+i \eta E_{A} .
\end{aligned}
$$

Write $E_{A, B}=X_{A, B} \exp \left(i \phi_{A, B}\right)$ and $\eta=\eta_{r}+i \eta_{i}=$ $|\eta| \exp (i \theta)$. Then, written in terms of amplitude and phase of $\eta$, Eqs. (A19) and (A20) become

$$
\begin{gathered}
\frac{d X_{A, B}}{d t}=\Gamma \frac{c}{2 n_{g}} a_{\mathrm{diff}}\left(N_{A, B}-N_{A, B t h}\right) X_{A, B} \\
-|\eta| X_{B, A} \sin (\theta \pm \phi), \\
\frac{\phi_{A, B}}{d t}=-\alpha_{H} \Gamma \frac{c}{2 n_{g}} a_{\mathrm{diff}}\left(N_{A, B}-N_{A, B t h}\right) \\
+\left(\omega-\Omega_{A, B}\right)+|\eta| \frac{X_{B, A}}{X_{A, B}} \cos (\theta \pm \phi),
\end{gathered}
$$

where $\phi=\phi_{B}-\phi_{A}$. Equations (A22) can be combined as

$$
\begin{aligned}
\frac{d \phi}{d t}= & \alpha_{H} \Gamma \frac{c}{2 n_{g}} a_{\mathrm{diff}}\left(N_{A}-N_{A t h}-N_{B}+N_{B t h}\right)-\Delta \Omega \\
& +|\eta|\left[\frac{X_{A}}{X_{B}} \cos (\theta-\phi)-\frac{X_{B}}{X_{A}} \cos (\theta+\phi)\right], \quad
\end{aligned}
$$

where $\Delta \Omega=\Omega_{B}-\Omega_{A}$ is the detuning between the cavity resonances of the two lasers.

The rate equations for carrier concentrations $N_{A}, N_{B}$ are

$$
\begin{aligned}
\frac{d N_{A, B}}{d t}= & P_{A, B}-\frac{N_{A, B}}{\tau_{N}} \\
& -\frac{c}{n}\left[g_{A, B t h}+a_{\mathrm{diff}}\left(N_{A, B}-N_{A, B t h}\right)\right] X_{A, B}^{2},
\end{aligned}
$$

where $P_{A, B}$ is the pumping rate (dimensions $L^{-3} T^{-1}$ ) and $\tau_{N}$ is the carrier lifetime. Note that so far the variables are not normalized, so that the dimensions of $N_{A}, N_{B}$ are $L^{-3}$, the fields have dimensions $L^{-3 / 2}$, and the gain and loss have dimension $L^{-1}$. Now define the dimensionless variables:

$$
\begin{gathered}
M_{A, B}=1+\frac{c}{n_{g}} \Gamma a_{\mathrm{diff}} \tau_{p}\left(N_{A, B}-N_{A, B t h}\right), \\
Q_{A, B}=1+\frac{c}{n_{g}} \Gamma a_{\mathrm{diff}} \tau_{p}\left(P_{A, B} \tau_{N}-N_{A, B t h}\right), \\
Y_{A, B}=\sqrt{\frac{c a_{\mathrm{diff}} \tau_{N}}{n}} X_{A, B} .
\end{gathered}
$$

In terms of these variables, Eqs. (A21), (A23), (A24) become

$$
\begin{gathered}
\frac{d Y_{A, B}}{d t}=\frac{1}{2 \tau_{p}}\left(M_{A, B}-1\right) Y_{A, B}-|\eta| Y_{B, A} \sin (\theta \pm \phi), \\
\frac{d \phi}{d t}=\frac{\alpha_{H}}{2 \tau_{p}}\left(M_{A}-M_{B}\right)-\Delta \Omega \\
\quad+|\eta|\left[\frac{Y_{A}}{Y_{B}} \cos (\theta-\phi)-\frac{Y_{B}}{Y_{A}} \cos (\theta+\phi)\right], \\
\frac{d M_{A, B}}{d t}=\frac{1}{\tau_{N}}\left[Q_{A, B}-M_{A, B}\left(1+Y_{A, B}^{2}\right)\right] .
\end{gathered}
$$

\section{APPENDIX B: LINEAR STABILITY ANALYSIS}

We write

$$
\begin{aligned}
Y_{A, B} & =Y_{A s, B s}+y_{A, B} e^{\lambda t}, \\
M_{A, B} & =M_{A s, B s}+m_{A, B} e^{\lambda t}, \quad \phi=\phi_{s}+\Delta \phi e^{\lambda t},
\end{aligned}
$$

where $y_{A, B} \ll Y_{A s, B s}, m_{A, B} \ll M_{A s, B s}$, and $\Delta \phi \ll \phi_{s}$. Substituting these expressions into Eqs. (18)-(21), and neglecting terms higher than first order, yields

$$
\begin{gathered}
2 \tau_{p} y_{A} \lambda=m_{A} Y_{A s}+2 \tau_{p}\left(y_{A}-y_{B}\right)\left(\eta_{r} \sin \phi_{s}+\eta_{i} \cos \phi_{s}\right) \\
-2 \tau_{p} Y_{B s} \Delta \phi\left(\eta_{r} \cos \phi_{s}-\eta_{i} \sin \phi_{s}\right), \\
2 \tau_{p} y_{B} \lambda=m_{B} Y_{B s}+2 \tau_{p}\left(y_{A}-y_{B}\right)\left(\eta_{r} \sin \phi_{s}-\eta_{i} \cos \phi_{s}\right) \\
+2 \tau_{p} Y_{A s} \Delta \phi\left(\eta_{r} \cos \phi_{s}+\eta_{i} \sin \phi_{s}\right), \\
\Delta \phi\left(\lambda-2 \eta_{i} \cos \phi_{s}\right)=\frac{\alpha_{H}}{2 \tau_{p}}\left(m_{A}-m_{B}\right)+2 \eta_{r} \cos \phi_{s} \frac{\left(y_{A}-y_{B}\right)}{Y_{s}}, \\
\tau_{N} m_{A} \lambda=-2 M_{A s} Y_{A s} y_{A}-m_{A}\left(1+Y_{A s}^{2}\right), \\
\tau_{N} m_{B} \lambda=-2 M_{B s} Y_{B s} y_{B}-m_{B}\left(1+Y_{B s}^{2}\right) .
\end{gathered}
$$

To proceed further we make the simplifying approximation that $M_{A s}=M_{B s} \cong 1$ (which is valid for $\left.\left|\tau_{p} \eta_{i}\right| \ll 1,\left|\tau_{p} \eta_{r}\right| \ll 1\right)$ and $Y_{A s}=Y_{B s} \cong Y_{s}$, with $Y_{s}^{2}=Q-1$. Then, adding and subtracting pairs of equations (B2) and (B3), and (B5) and (B6), gives

$$
\begin{gathered}
2 \tau_{p} \lambda\left(y_{A}+y_{B}\right)-4 \tau_{p} \eta_{r} \sin \phi_{s}\left(y_{A}-y_{B}\right) \\
=\left(m_{A}+m_{B}\right) Y_{s}+4 \tau_{p} Y_{s} \Delta \phi \eta_{i} \sin \phi_{s}, \\
\left(m_{A}+m_{B}\right)\left(\tau_{N} \lambda+1+Y_{s}^{2}\right)=-2 M_{s} Y_{s}\left(y_{A}+y_{B}\right), \\
2 \tau_{p}\left(y_{A}-y_{B}\right)\left(\lambda-2 \eta_{i} \cos \phi_{s}\right) \\
=\left(m_{A}-m_{B}\right) Y_{s}-4 \tau_{p} Y_{s} \eta_{r} \Delta \phi \cos \phi_{s}, \\
\left(m_{A}-m_{B}\right)\left(\tau_{N} \lambda+1+Y_{s}^{2}\right)=-2 M_{s} Y_{s}\left(y_{A}-y_{B}\right) .
\end{gathered}
$$

For the case of zero detuning $(\Delta \Omega=0)$ the phase shift between the modes $\phi_{s}$ is 0 or $\pi$, and hence Eq. (B7) simplifies to

$$
2 \tau_{p} \lambda\left(y_{A}+y_{B}\right)=\left(m_{A}+m_{B}\right) Y_{s} .
$$
form

Equations (B8) and (B11) give a quadratic equation of the

$$
\lambda^{2}+B_{1} \lambda+B_{2}=0
$$

where the coefficients $B_{i}$ are given by

$$
\begin{gathered}
B_{1}=\frac{1+Y_{s}^{2}}{\tau_{N}}=\frac{Q}{\tau_{N}}, \\
B_{2}=\frac{M_{s} Y_{s}^{2}}{\tau_{N} \tau_{p}}=\frac{Q-1}{\tau_{N} \tau_{p}} .
\end{gathered}
$$


Writing $\lambda=\gamma_{D}+i \omega_{R}$, the solution of Eq. (B12) is

$$
\gamma_{D}=-\frac{Q}{2 \tau_{N}}, \quad \omega_{R}^{2}=\frac{Q-1}{\tau_{N} \tau_{p}}-\gamma_{D}^{2}
$$

We recognize $\omega_{R}$ as the angular frequency, and $\gamma_{D}$ as the associated damping rate, of the relaxation oscillations.

Equations (B4), (B9), and (B10) give a cubic equation of the form

$$
\lambda^{3}+A_{1} \lambda^{2}+A_{2} \lambda+A_{3}=0,
$$

where the coefficients $A_{i}$ are given by

$$
\begin{gathered}
A_{1}=\frac{1+Y_{s}^{2}}{\tau_{N}}-4 \eta_{i} \cos \phi_{s} \\
A_{2}=4|\eta|^{2} \cos ^{2} \phi_{s}-4 \eta_{i} \cos \phi_{s} \frac{1+Y_{s}^{2}}{\tau_{N}}+\frac{M_{s} Y_{s}^{2}}{\tau_{N} \tau_{P}} \\
A_{3}=4|\eta|^{2} \cos ^{2} \phi_{s} \frac{1+Y_{s}^{2}}{\tau_{N}}-2 \frac{M_{s} Y_{s}^{2}}{\tau_{N} \tau_{p}} \cos \phi_{s}\left(\eta_{i}+\alpha_{H} \eta_{r}\right) .
\end{gathered}
$$

Equations (33)-(35) are equivalent to Eqs. (4.4.31)(4.4.33) in [37], although there the detuning between lasers was ignored $(\Delta \Omega=0)$ and hence the phase shift between the modes $\phi_{s}$ was 0 or $\pi$.

The conditions for stable steady-state solutions of Eq. (B16) are

$$
A_{1}>0, \quad A_{3}>0, \quad A_{1} A_{2}-A_{3}>0 .
$$

Substituting in Eq. (B19) for $M_{s}$ and $Y_{s}$ yields for the second condition of Eq. (B20)

$$
2 Q|\eta|^{2} \cos ^{2} \phi_{s}>\frac{(Q-1)}{\tau_{p}} \cos \phi_{s}\left(\eta_{i}+\alpha_{H} \eta_{r}\right)
$$

For zero detuning, $\Delta \Omega=0$, substituting $\phi_{s}=0$ and $\phi_{s}=\pi$ yields Eqs. (32) and (34), respectively.
In the general case of nonzero detuning, from Eq. (B21) the two possible conditions for stability are

$$
\begin{aligned}
\cos \phi_{s}> & \frac{\left(\eta_{i}+\alpha_{H} \eta_{r}\right)}{|\eta|^{2}} \frac{(Q-1)}{2 Q \tau_{p}}, \\
& \cos \phi_{s}>0 .
\end{aligned}
$$

For parameter values of interest $\left|\tau_{p} \eta_{i}\right| \ll 1,\left|\tau_{p} \eta_{r}\right| \ll 1$, and hence Eq. (B22) gives values of $\cos \phi_{s}$ that are greater than 1. It follows that the solution of interest must be Eq. (B23), which leads to

$$
\left|\sin \phi_{s}\right|<1 \text {. }
$$

From Eq. (29), this gives Eq. (30).

We consider now the third stability condition of Eq. (B20). Substituting the expressions from Eqs. (B17)-(B19), this gives

$$
\begin{aligned}
& {\left[\frac{1+Y_{s}^{2}}{\tau_{N}}-4 \eta_{i} \cos \phi_{s}\right]} \\
& \quad \times\left[4|\eta|^{2} \cos ^{2} \phi_{s}-4 \eta_{i} \cos \phi_{s} \frac{1+Y_{s}^{2}}{\tau_{N}}+\frac{M_{s} Y_{s}^{2}}{\tau_{N} \tau_{P}}\right] \\
& >4|\eta|^{2} \cos ^{2} \phi_{s} \frac{1+Y_{s}^{2}}{\tau_{N}}-2 \frac{M_{s} Y_{s}^{2}}{\tau_{N} \tau_{p}} \cos \phi_{s}\left(\eta_{i}+\alpha_{H} \eta_{r}\right) .
\end{aligned}
$$

Substituting for $M_{s}$ and $Y_{s}$ as before, the boundary condition can be rewritten as

$$
\begin{aligned}
& (Q-1)\left[Q-2 \tau_{N}\left(\eta_{i}-\alpha_{H} \eta_{r}\right) \cos \phi_{s}\right]-4 \eta_{i} \tau_{p} \cos \phi_{s} \\
& \quad \times\left(Q^{2}-4 \eta_{i} \tau_{N} Q \cos \phi_{s}+4 \tau_{N}^{2}|\eta|^{2} \cos ^{2} \phi_{s}\right)>0 .
\end{aligned}
$$

For zero detuning, $\Delta \Omega=0$, substituting $\phi_{s}=0$ and $\phi_{s}=\pi$ into Eq. (B26) yields Eqs. (33) and (35), respectively.

In the general case of nonzero detuning, substituting for $\phi_{s}$ from Eq. (29) in Eq. (B26) gives the region of stability. However, for parameter values of interest $\left|\tau_{p} \eta_{i}\right| \ll 1$, and hence to a very good level of approximation the last 3 terms on the left-hand side of Eq. (B26) can be neglected in comparison to the first 2 terms. It follows that

$$
Q-2 \tau_{N}\left(\eta_{i}-\alpha_{H} \eta_{r}\right) \cos \phi_{s}>0 .
$$

From Eq. (29), this gives Eq. (31).
[1] J. E. Ripper and T. L. Paoli, Appl. Phys. Lett. 17, 371 (1970).

[2] Edited by D. Botez and D. R. Scifres, Diode Laser Arrays (Cambridge University Press, Cambridge, 1994).

[3] N. W. Carlsson, Monolithic Diode-Laser Arrays (SpringerVerlag, Berlin, 1994).

[4] E. Marom, O. G. Ramer, and S. Ruschin, IEEE J. Quantum Electron. 20, 1311 (1984).

[5] T. Czyszanowski, R. P. Sarzała, M. Dems, J. Walczak, M. Wasiak, W. Nakwaski, V. Iakovlev, N. Volet, and E. Kapon, IEEE J. Sel. Top. Quantum Electron. 19, 1702010 (2013).

[6] C. Sigler, C.-C. Chang, J. D. Kirch, L. J. Mawst, D. Botez, and T. Earles, IEEE J. Sel. Top. Quantum Electron. 21, 1200810 (2015).
[7] S. S. Wang and H. G. Winful, Appl. Phys. Lett. 52, 1774 (1988).

[8] H. G. Winful and S. S. Wang, Appl. Phys. Lett. 53, 1894 (1988).

[9] R. F. M. Hendriks, M. P. van Exter, J. P. Woerdman, and C. J. van der Poel, Appl. Phys. Lett. 69, 869 (1996).

[10] A. Yariv, Optical Electronics in Modern Communications, 5th ed. (Oxford University Press, New York, 1997), Sec. 13.8.

[11] J. Katz, E. Kapon, C. Lindsey, S. Margalit, and A. Yariv, Appl. Opt. 23, 2231 (1984).

[12] J. K. Butler, D. E. Ackley, and M. Ettenberg, IEEE J. Quantum Electron. QE-21, 458 (1985).

[13] G. A. Wilson, R. K. DeFreez, and H. G. Winful, IEEE J. Quantum Electron. 27, 1696 (1991). 
[14] P. Ru, P. K. Jakobsen, J. V. Moloney, and R. A. Indik, J. Opt. Soc. Am. B 10, 507 (1993).

[15] G. Bouwmans, B. Segard, D. Dangoisse, and P. Glorieux, J. Opt. Soc. Am. B 17, 781 (2000).

[16] H. Laabs and B. Ozygus, Optics and Laser Technol. 29, 401 (1997).

[17] H. Erzgräber, S. Wieczorek, and B. Krauskopf, Phys. Rev. E 78, 066201 (2008).

[18] M. B. Spencer and W. E. Lamb, Jr., Phys. Rev. A 5, 884 (1972).

[19] M. B. Spencer and W. E. Lamb, Jr., Phys. Rev. A 5, 893 (1972).

[20] J. Xu, K. K. Lee, and Y. C. Chen, Opt. Commun. 117, 198 (1995).

[21] M. Leonés, H. Lamela, and G. Carpintero, Proc. SPIE 3625, 707 (1999).

[22] H. Lamela, M. Leonés, G. Carpintero, C. Simmendinger, and O. Hess, IEEE J. Sel. Top. Quantum Electron. 7, 192 (2001).

[23] A. E. Siegman, J. Opt. Soc. Am. A 20, 1617 (2003).

[24] W. O. Schlosser, Bell Syst. Tech. J. 52, 887 (1973).

[25] J. Buus, Conference Proceedings: 7th European Microwave Conference, Copenhagen, 1977 (Microwave Exhibitions and Publishers, Sevenoaks, 1977), pp. 29-33.

[26] J. Buus and D. Botez, IEEE Photon. Technol. Lett. 8, 92 (1996).

[27] S. Yanchuk, K. R. Schneider, and L. Recke, Phys. Rev. E 69, 056221 (2004).
[28] X. Wang, C. Xiong, and J. Luo, Opt. Commun. 282, 382 (2009).

[29] E. J. Doedel, A. R. Champneys, T. Fairgrieve, Y. Kuznetsov, B. Oldeman, R. Pfaffenroth, B. Sandastede, X. Wang, and C. Zhang, AUTO-07p: Continuation and Bifurcation Software for Ordinary Differential Equations (Concordia University, Montreal, 2008).

[30] J. Sieber, SIAM J. Appl. Dyn. Syst. 2, 248 (2002).

[31] S. Wieczorek, B. Krauskopf, T. B. Simpson, and D. Lenstra, Phys. Rep. 416, 1 (2005).

[32] P. Hamel, S. Haddadi, F. Raineri, P. Monnier, G. Beaudoin, I. Sagnes, A. Levenson, and A. M. Yacomotti, Nat. Photon. 9, 311 (2015).

[33] E. Clerkin, S. O’Brien, and A. Amann, Phys. Rev. E 89, 032919 (2014).

[34] Y. A. Kuznetsov, Elements of Applied Bifurcation Theory, 3rd ed. (Springer, New York, 2004).

[35] M. Marconi, J. Javaloyes, F. Raineri, J. A. Levenson, and A. M. Yacometti, Opt. Lett. 41, 5628 (2016).

[36] M. T. Johnson, D. F. Siriani, M. P. Tan, and K. D. Choquette, Appl. Phys. Lett. 103, 201115 (2013).

[37] G. A. Wilson, Ph.D. thesis, Picosecond dynamics of twin-emitter coherent semiconductor laser arrays, Oregon Graduate Institute of Science and Technology, 1992. 